\title{
Determination of pion and kaon fragmentation functions including spin asymmetries data in a global analysis
}

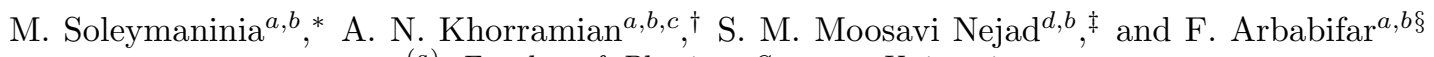 \\ (a) Faculty of Physics, Semnan University, \\ 35131-19111 Semnan, Iran \\ (b) School of Particles and Accelerators, \\ Institute for Research in Fundamental Sciences (IPM), \\ P.O.Box 19395-5531, Tehran, Iran \\ ${ }^{(c)}$ Stanford Institute for Theoretical Physics and Department of Physics, \\ Stanford University, Stanford, CA 94305-4060, USA \\ (d) Faculty of Physics, Yazd University, \\ P.O.Box 89195-741, Yazd, Iran
}

(Dated: September 12, 2018)

\begin{abstract}
We present new functional form of pion and kaon fragmentation functions up to next-to-leading order obtained through a global fit to single-inclusive electron-positron annihilation data and also employ, the semi-inclusive deep inelastic scattering asymmetry data from HERMES and COMPASS to determine fragmentation functions. Also we apply very recently electron-positron annihilation data from BaBar and Belle at $\sqrt{s}=10.54 \mathrm{GeV}$ and $\sqrt{s}=10.52 \mathrm{GeV}$, respectively. In this analysis we consider the impression of semi-inclusive deep inelastic scattering asymmetry data on the fragmentation functions, where the produced hadrons of different electric charge are identified. We break symmetry assumption between quark and anti-quark fragmentation functions for favored partons by using the asymmetry data. The results of our analysis are in good agreement with electronpositron annihilation data and also with all the semi-inclusive deep inelastic scattering asymmetry data. Also we apply the obtained fragmentation functions to predict the scaled-energy distribution of $\pi^{+} / K^{+}$inclusively produced in top-quark decays at next-to-leading order using the zero-mass variable-flavor-number scheme exploiting the universality and scaling violations of fragmentation functions.

PACS numbers: 13.87.Fh, 13.66.Bc, $13.60 . \mathrm{Hb}, 13.85 \mathrm{Ni}$
\end{abstract}

\section{INTRODUCTION}

In high energy processes at Relativistic Heavy Ion Collider (RHIC) and Large Hadron Collider (LHC), the quantum chromodynamics (QCD) predictions of cross sections have very important role. In the general case the parton distribution functions (PDFs) of initial hadrons, parton-level differential cross sections, and fragmentation functions are three necessary ingredients to calculate cross sections. Fragmentation functions (FFs) form the non-perturbative component of the hard scattering process and describe the transition of a parton into the outgoing hadron.

The importance of FFs is for model independent predictions of the cross sections at LHC in which a hadron is detected in the outgoing productions depend on FFs. Interest of FFs is found for example in tests of QCD such as theoretical calculations for recent measurements of inclusive production in proton-proton collisions at RHIC, and in investigating the origin of the proton spin. In the naive

\footnotetext{
*Electronic address: Maryam soleymaninia@ipm.ir

${ }^{\dagger}$ Electronic address: Khorramiana@theory.ipm.ac.ir

‡Electronic address: Mmoosavi@yazduni.ac.ir

§Electronic address: Farbabifar@ipm.ir
}

parton model the FFs are independent of the factorization scale (depending only on the scaling variable $z$ ) but in QCD-improved parton model the scaling violations of FFs remain to be subject to Dokshitzer- Gribov-LipatovAlteralli-Parisi (DGLAP) evolution equations [1]. Note that FFs can be extracted from fits to data at intermediate to large momentum fractions.

FFs are studied in electron-positron annihilation, leptonhadron and hadron-hadron scattering processes. Among all, the $e^{+} e^{-}$annihilation provides a clean environment to determine the fragmentation densities, but without an initial hadron state one can not separate a quark from an anti-quark distributions. Since the most precise data from $e^{+} e^{-}$annihilation exists for the production of the lightest charged hadrons (pion, kaon and proton), we are interested in the fragmentation processes of the partons into the pion and kaon in electron-positron annihilation and semi-inclusive deep inelastic scattering. Fragmentation functions are included in hadron-production processes in electron-positron annihilation, lepton-proton or nucleus scattering, proton-proton and heavy ions collisions. Such processes are important in hadron physics for studying and investigating the origin of the proton spin [2, 3] and in reported results in Refs. [4 7], the role of FFs to determine the polarized parton distribution functions is pointed out. In the present analysis to determine FFs, we consider semi-inclusive deep inelastic scattering 
(SIDIS) asymmetry data along with the data on the $e^{+} e^{-}$ annihilation from LEP (ALEPH, DELPHI and OPAL collaborations), SLAC (SLD and TPC collaborations), DESY (TASSO collaboration), KEK (TOPAZ collaboration) [8 -15] and very recent $\mathrm{BaBar}$ and Belle data at the SLAC and the KEKB [16, 17], respectively. There are also some other kinds of new analysis performed by using these new reported data [18, 19].

There are already several theoretical studies on QCD analysis for FFs which are listed in [20], in particular, used parameterizations were widely obtained by KKP and AKK collaborations [21] and Kretzer [22]. As it is shown in Ref. [23], there are differences between the fragmentation functions of KKP and Kretzer, therefore it is convenient to make an error calculation for the fragmentation functions to check the consistency of results. In this regard, an attempt for determining the FFs and their uncertainties has been already done in HKNS and DSS collaborations [23, 24]. But we have an opportunity to use the asymmetry SIDIS experimental data from HERMES [25] and COMPASS [26, 27] which is the first attempt to study how much the asymmetry SIDIS data are effective for determination of FFs and also their uncertainties.

Since the decay of top-quark is one of the interesting subjects at LHC we shall make our theoretical predictions for the energy spectrum of $\pi^{+}$- and $K^{+}$-mesons produced in top decay using the FFs obtained from our present work. We will also compare our predictions with the known results of Refs. [23, 24, 28].

This paper is organized as follows. In Section [I] we explain the hadronization process in electron-positron collision by introducing the FFs. Double spin asymmetry in semi-inclusive deep inelastic scattering processes is defined in this section too. In section [II] we describe our formalism and parametrization form for pion and kaon fragmentation densities. The global $\chi^{2}$ minimization for data is defined in this section. We also outline the Hessian method for assessing the neighborhood of global minimum. In section IV our formalism to predict the energy distribution of $\pi^{+}$and $K^{+}$in top-quark decay is explained. The full results for pion and kaon FFs and their uncertainties are listed in section $\mathrm{V}$, Comparison of our results with experimental data and the other models and also our predictions of energy spectrum of outgoing pion and kaon in top-quark decay are presented in this section. Our conclusion is given in section VI.

\section{THEORETICAL FORMALISM FOR FRAGMENTATION FUNCTIONS}

The fragmentation functions are related to the low energy components of the hadron production processes and they form the non-perturbative aspect of QCD. FFs describe the inclusive emission of a hadron from a parton and they cannot be precisely calculated by theoretical methods at this stage. The perturbative QCD frame- work is used to study single-inclusive hadron production in $e^{+} e^{-}$annihilation, lepton-nucleon DIS, and hadronhadron collisions, where the factorization theorem is a strong tool to study such processes. This theorem states that the cross section can be expressed in terms of perturbatively calculable partonic hard-scattering cross sections, PDFs and FFs in which the two last are related to the low energy components of QCD processes. The low energy components of QCD processes are universal and they can be used to make predictions.

In this section, we explain the theoretical framework relevant for our global QCD analysis of fragmentation functions.

\section{A. Single-inclusive $e^{+} e^{-}$annihilation}

Since, in the single-inclusive $e^{+} e^{-}$annihilation processes

$$
e^{+} e^{-} \rightarrow(\gamma, Z) \rightarrow H+X
$$

one should not deal with the uncertainty introduced by PDFs in comparison with the hadron collisions, then the optimal way to determine FFs is to fit them to experimental date extracted from these processes. In the above process, $X$ stands for the unobserved jets which are produced along with a detected hadron $H$.

The perturbative QCD framework is used to study hadron production in $e^{+} e^{-}$annihilation, where the factorization theorem is an important tool to study this process. According to the hard-scattering factorization theorem of the parton model [29, 30], the cross section can be written as a sum of convolutions of perturbatively calculable the partonic hard scattering cross sections $d \sigma_{a}\left(y, \mu_{R}, \mu_{F}\right) / d y[22,31,32]$ with the non-perturbative fragmentation functions of the hadron $H$ from a parton $a, D_{a}^{H}\left(x, \mu_{F}\right)$ as following

$$
\frac{1}{\sigma_{t o t}} \frac{d \sigma^{H}}{d z}=\sum_{a} \int_{z}^{1} \frac{d y}{y} D_{a}^{H}\left(\frac{z}{y}, \mu_{F}\right) \frac{1}{\sigma_{t o t}} \frac{d \sigma_{a}}{d y}\left(y, \mu_{R}, \mu_{F}\right),
$$

where the $a$ stands for one of the partons $a=$ $g, u, \bar{u}, \cdots, b, \bar{b}$. We denote the four-momenta of the intermediate gauge boson and the $H$ hadron by $q$ and $p_{H}$, respectively, so that $s=q^{2}$ and the scaling variable $z$ is defined as $z=2\left(p_{H} . q\right) / q^{2}$. In the center-ofmass (c.m.) frame, $z$ is simplified to $z=2 E_{H} / \sqrt{s}$ which refers to the energy of $H$ scaled to the beam energy. The function $D_{a}^{H}\left(x, \mu_{F}\right)$ indicates the probability to find the hadron $H$ from a parton $a$ with the scaled energy fraction $x$. In equation above, $y$ is defined in analogy to $z$ as $y=2\left(p_{a} . q\right) / q^{2}$, where $p_{a}$ is the four-momentum of parton $a$. The renormalization and factorization scales are given by $\mu_{R}$ and $\mu_{F}$, respectively, however one can choose two different values for them but a choice often made consists of setting $\mu_{R}^{2}=\mu_{F}^{2}=Q^{2}$ and we shall 
adopt this convention in this work.

At NLO, the total cross section is described by the $q \bar{q}-$ pair creation subprocesses, $e^{+} e^{-} \rightarrow(\gamma, Z) \rightarrow q \bar{q}+(g)$, as

$\sigma_{\text {tot }}=N_{c} \sigma_{0} \sum_{i=1}^{n_{f}}\left(V_{q_{i}}^{2}+A_{q_{i}}^{2}\right)\left[1+\frac{\alpha_{s}(\mu)}{2 \pi} C_{F} \frac{3}{2}+\mathcal{O}\left(\alpha_{s}^{2}\right)\right]$,

where $C_{F}=\left(N_{c}^{2}-1\right) /\left(2 N_{c}\right)=4 / 3$ for $N_{c}=3$ quark colors and $\sigma_{0}=\left(4 \pi \alpha^{2} / 3 s\right)$ is the leading order total cross section of $e^{+} e^{-} \rightarrow \mu^{+} \mu^{-}$for massless muons, $n_{f}$ is the number of active flavors, $N_{c}=3$ is the number of quark colors and $\alpha$ is the electro-weak coupling constant. $V_{q_{i}}$ and $A_{q_{i}}$ are the effective vector and axial-vector couplings of quark $q_{i}$ to the both intermediate photon and $Z$-boson, which can be found in Ref. [33]. For small energies, $\sqrt{s} \ll M_{Z}$, for the summation of squared effective electro-weak charges one has $V_{q_{i}}^{2}+A_{q_{i}}^{2}=e_{q_{i}}^{2}$ where $e_{q_{i}}$ is the electric charge of quark $q_{i}$, see Ref. [33].

In our global analysis of FFs, some of the data sets from the OPAL and the ALEPH experiments are in the form of $1 / N_{Z \rightarrow \text { Hadron }}\left(d N^{H} / d z\right)$ where $N$ is the number of detected events. This is defined as the ratio of the singleinclusive $e^{+} e^{-}$annihilation cross section (2) in a certain bin of $z$ to the totally inclusive rate, i.e.

$$
\frac{1}{N_{\text {tot }}} \frac{d N^{H}}{d z} \equiv \frac{1}{\sigma_{\text {tot }}} \frac{d \sigma^{H}}{d z} .
$$

\section{B. Hadronization process in ep collisions and spin asymmetry}

Generally parton distribution function $q\left(x, Q^{2}\right)$ expresses the probability density to find a parton $q$ in a nucleon carrying fraction $x$ of target nucleon momentum at the transfer momentum $Q^{2}$. For the polarized PDFs, we assume that a proton is made of massless partons with the positive and negative helicity distributions and thus the difference

$$
\delta q\left(x, Q^{2}\right)=q_{+}\left(x, Q^{2}\right)-q_{-}\left(x, Q^{2}\right),
$$

demonstrates how much the parton of flavor $q$ represents of the proton polarization. Universally, the functional forms of the polarized and unpolarized PDFs are determined by a QCD fit to the experimental data obtained from various interactions. These analysis have been discussed in lots of recent reviews 2, 3, 34 52 and new more precise investigations are still in progress.

In order to cover more kinematics region in the current analysis we consider polarized SIDIS process, $\vec{l}(l)+$ $\vec{N}\left(p_{N}\right) \rightarrow l^{\prime}\left(l^{\prime}\right)+H\left(p_{H}\right)+X$, where hadron $H$ is also detected along with the scattered lepton $l^{\prime}$ and jets $X$. This process gives a remarkable information concerning the nucleon structure in quite distinct kinematics which probes different aspects of fragmentation distributions.
Moreover, SIDIS data help us to specify the difference between the quark and anti-quark distributions in the nucleon considering outgoing produced hadrons which is not possible in fully inclusive experiments.

In the polarized SIDIS, the measured double spin asymmetry $A_{1}^{N, H}$ can be expressed in terms of the ratio of polarized and unpolarized structure functions $g_{1}^{N, H}$ and $F_{1}^{N, H}$ [43], as

$$
A_{1}^{N, H}\left(x, z, Q^{2}\right)=\frac{g_{1}^{N, H}\left(x, z, Q^{2}\right)_{N L O}}{F_{1}^{N, H}\left(x, z, Q^{2}\right)_{N L O}},
$$

where $z$ is the scaled energy fraction of the outgoing hadron, $Q^{2}$ is the transfer momentum and $x$ is the Bjorken scaling variable. The indices $N$ and $H$ stand for the different nucleon targets and outgoing detected hadron, respectively.

In the NLO approximation, the polarized and unpolarized structure functions $g_{1}^{N, H}$ and $F_{1}^{N, H}$ in SIDIS processes are presented as (see Ref. [43])

$$
\begin{aligned}
2 g_{1}^{N, H}\left(x, z, Q^{2}\right)= & \sum_{q, \bar{q}}^{n_{f}} e_{q}^{2}\left\{\Delta q\left(x, Q^{2}\right) D_{q}^{H}\left(z, Q^{2}\right)\right. \\
& +\frac{\alpha_{s}\left(Q^{2}\right)}{2 \pi}\left[\Delta q \otimes \Delta C_{q q}^{(1)} \otimes D_{q}^{H}\right. \\
& +\Delta q \otimes \Delta C_{g q}^{(1)} \otimes D_{g}^{H} \\
& \left.\left.+\Delta g \otimes \Delta C_{q g}^{(1)} \otimes D_{q}^{H}\right]\left(x, z, Q^{2}\right)\right\},
\end{aligned}
$$

and

$$
\begin{aligned}
2 F_{1}^{N, H}\left(x, z, Q^{2}\right)= & \sum_{q, \bar{q}}^{n_{f}} e_{q}^{2}\left\{q\left(x, Q^{2}\right) D_{q}^{H}\left(z, Q^{2}\right)\right. \\
& +\frac{\alpha_{s}\left(Q^{2}\right)}{2 \pi}\left[q \otimes C_{q q}^{(1)} \otimes D_{q}^{H}\right. \\
& +q \otimes C_{g q}^{(1)} \otimes D_{g}^{H} \\
& \left.\left.+g \otimes C_{q g}^{(1)} \otimes D_{q}^{H}\right]\left(x, z, Q^{2}\right)\right\},
\end{aligned}
$$

where $n_{f}$ is the number of active flavors, $e_{q}$ is the electric charge of quark $q, \alpha_{s}$ is the strong coupling constant, $\Delta q$ and $q$ are polarized and unpolarized parton densities and $\Delta C_{i j}^{(1)}(x, z)$ and $C_{i j}^{(1)}(x, z)$ are the polarized and unpolarized NLO Wilson coefficient functions, respectively, presented in Ref. [53]. The corresponding parton FFs, $D_{q, \bar{q}}^{H}$ and $D_{g}^{H}$, are determined in the present global analysis and play a significant role in determination of $A_{1}^{N, H}$. 
According to Eq. (6) the double spin asymmetry $A_{1}^{N, H}$ depends on polarized and unpolarized parton distribution functions so in order to calculate the double spin asymmetry, we need to use the results of available PPDFs and PDFs sets. Here we choose the latest DSSV PPDFs and KKT12 PDFs [3, 34], however, the different choices of PPDFs and PDFs do not change our result considerably.

\section{QCD ANALYSIS \& PARAMETRIZATION}

\section{A. ZM-VFN scheme}

In a parton fragmentation function $D_{i}^{H}\left(z, \mu^{2}\right), z$ represents the fraction of a parton's momentum carried by a produced hadron while in a parton distribution $q\left(x, \mu^{2}\right)$, $x$ represents the fraction of a hadron's momentum carried by constituent parton. In both cases, QCD parton model approach would predict $z$ and $x$-distributions independent of the factorization scale. Note that the similar violations of this scaling behavior are happened when QCD corrections are taken into account 29], in other words, beyond the leading order of perturbative QCD these universal functions are factorization-scale dependent. The $z$ dependence of the fragmentation functions is of non-perturbative aspect of QCD and they are not yet calculable from first principles. However, once they are given at the initial fragmentation scale $\mu_{0}$ their $\mu$ evolution is determined by the DGLAP renormalization group equations [1] which is very similar to those for parton densities. For example, the flavor-singlet evolution equation reads [54]

$$
\begin{aligned}
\frac{\partial}{\partial \ln \mu^{2}}\left(\begin{array}{c}
D_{S}^{H}\left(z, \mu^{2}\right) \\
D_{g}^{H}\left(z, \mu^{2}\right)
\end{array}\right)= & \left(\begin{array}{cc}
P_{q q}(z) & P_{g q}(z) \\
P_{q g}(z) & P_{g g}(z)
\end{array}\right) \\
& \otimes\left(\begin{array}{c}
D_{S}^{H}\left(z, \mu^{2}\right) \\
D_{g}^{H}\left(z, \mu^{2}\right)
\end{array}\right),
\end{aligned}
$$

where $D_{S}^{H}\left(z, \mu^{2}\right)$ refers to the singlet function, $D_{S}^{H}\left(z, \mu^{2}\right)=\sum_{q}\left[D_{q}^{H}\left(z, \mu^{2}\right)+D_{\bar{q}}^{H}\left(z, \mu^{2}\right)\right]$, and the convolution integral $\otimes$ is defined by

$$
f(z) \otimes g(z)=\int_{z}^{1} \frac{d y}{y} f(y) g\left(\frac{z}{y}\right) .
$$

The functions $P_{j i}$ are time-like splitting functions which are the same as those in deep inelastic scattering at the lowest order but the higher order terms are different. The third-order contributions $\left(\mathrm{N}^{2} \mathrm{LO}\right)$ to the quarkgluon and gluon-quark timelike splitting functions can be found in Ref. [54]. The evolution equations are essentially the same as the PDF case, so that the same numerical method can be used to obtain a solution. Also the flavor non-singlet evolution equation can be found in Refs. [5557].
To extract the fragmentation functions from data analysis there are several approaches and in the present analysis we adapt the zero-mass variable-flavor-number (ZMVFN) scheme [58]. In this scheme, all quarks are treated as massless particles and the non-zero values of the charm and bottom-quark masses only enter through the initial conditions of the FFs. The number of active flavors also varies with the factorization scale where for the scales higher than the respective flavor thresholds, the quark is active as a parton. This scheme works best for high energy scales, where $m_{Q}=0$ is a good approximation.

We also evaluate $\alpha_{s}^{\left(n_{f}\right)}(\mu)$ at NLO in the improvedminimal subtraction $(\overline{\mathrm{MS}})$ scheme using

$$
\alpha_{s}^{\left(n_{f}\right)}(\mu)=\frac{1}{b_{0} \log \left(\mu^{2} / \Lambda^{2}\right)}\left\{1-\frac{b_{1} \log \left[\log \left(\mu^{2} / \Lambda^{2}\right)\right]}{b_{0}^{2} \log \left(\mu^{2} / \Lambda^{2}\right)}\right\},
$$

with

$$
b_{0}=\frac{33-2 n_{f}}{12 \pi}, \quad b_{1}=\frac{153-19 n_{f}}{24 \pi^{2}},
$$

where $\Lambda$ is the typical QCD scale. We adopt $\Lambda_{Q C D}^{(4)}=$ $334.0 \mathrm{MeV}$ [59] for NLO adjusted such that $\alpha_{s}^{(5)}=0.1184$ for $M_{Z}=91.1876 \mathrm{GeV}$ [60]. Also $\Lambda_{Q C D}^{(4)}$ at LO is fixed in our fits to $220.0 \mathrm{MeV}$.

\section{B. Parametrization of fragmentation functions}

We parameterize the pion and kaon fragmentation functions at LO and NLO considering the single inclusive annihilation (SIA) and SIDIS data. At the initial scale $\mu_{0}$ this parametrization contains a functional form as

$$
D_{i}^{H}\left(z, \mu_{0}^{2}\right)=N_{i} z^{\alpha_{i}}(1-z)^{\beta_{i}}\left[1-e^{-\gamma_{i} z}\right],
$$

which is a convenient form for the light hadrons. A simple polynomial parametrization with just 3 parameters $N_{i}, \alpha_{i}$ and $\beta_{i}$ controls the small and large $z$ region [61]. Accordingly, power term in $z$ emphasizes the small $z$ region and power term in $(1-z)$ restricts the large $z$ region. We consider the extra term $\left[1-e^{-\gamma_{i} z}\right]$ to control medium $z$ region and to improve the accuracy of the global fit 62 ]. The free parameters $N_{i}, \alpha_{i}, \beta_{i}$ and $\gamma_{i}$ are determined by fitting $\chi^{2}$ of SIA and SIDIS data. In the $\overline{M S}$ scheme, there is an important sum rule of the FFs on the energy conservation as

$$
\int_{0}^{1} d z z \sum_{H} D_{i}^{H}\left(z, \mu^{2}\right)=1
$$

which means that each parton surly will fragment into some hadron $H$. Since the summation over all the hadrons can not be taken practically and the behavior of small $z$ is unstable, Eq. (14) can not be a viable constraint in a global analysis. 
The initial scale $\mu_{0}$ is different for partons. The starting scale for the FFs of the light-quarks $(u / \bar{u}, d / \bar{d}, s / \bar{s})$ and $g$ into $\pi^{ \pm} / K^{ \pm}$-mesons is $\mu_{0}^{2}=1 \mathrm{GeV}^{2}$ and it is taken at $\mu_{0}^{2}=m_{c}^{2}$ and $\mu_{0}^{2}=m_{b}^{2}$ for charm and bottom-quarks [63, 64]. We choose $m_{c}=1.43 \mathrm{GeV}$ and $m_{b}=4.3 \mathrm{GeV}$ in our analysis. Then these FFs are evolved to higher scales using the DGLAP group equations in Eq. (91).

In all analysis it is necessary to use different assumptions for various FFs. In our analysis, we take the same fragmentation densities for valence quarks. Since the possibility of $\pi^{+} / K^{+}$-production from valence or favored quarks is more than sea or unfavored quarks then we assume distinct fragmentation functions for the light sea quarks. Because of mass difference, the different functions are also specified for heavy quarks and we assume the same FFs for heavy quark and its anti-quark like $D_{c}^{H}=D_{\bar{c}}^{H}$ and $D_{b}^{H}=D_{\bar{b}}^{H}$. According to the pion structure $\left|\pi^{+}\right\rangle=|u \bar{d}\rangle$ and the general functional form presented in Eq. (13), for the parton-FFs into $\pi^{+}$one has

$$
\begin{aligned}
D_{u, \bar{d}}^{\pi^{+}} \quad\left(z, \mu_{0}^{2}\right) & =N_{u}^{\pi^{+}} z^{\alpha_{u}^{\pi^{+}}}(1-z)^{\beta_{u}^{\pi^{+}}}\left[1-e^{-\gamma_{u}^{\pi^{+}} z}\right], \\
D_{d, \bar{u}, s, \bar{s}}^{\pi^{+}} & \left(z, \mu_{0}^{2}\right)=N_{d}^{\pi^{+}} z^{\alpha_{d}^{\pi^{+}}}(1-z)^{\beta_{d}^{\pi^{+}}}\left[1-e^{-\gamma_{d}^{\pi^{+}} z}\right],
\end{aligned}
$$

where we impose $S U(2)$ isospin invariance between $u$ and $\bar{d}$-quarks for pion. Isospin symmetry is also considered for sea quarks of pion fragmentation functions. Gluon and heavy quarks FFs are defined as

$$
\begin{aligned}
& D_{g}^{\pi^{+}} \quad\left(z, \mu_{0}^{2}\right)=N_{g}^{\pi^{+}} z^{\alpha_{g}^{\pi^{+}}}(1-z)^{\beta_{g}^{\pi^{+}}}\left[1-e^{-\gamma_{g}^{\pi^{+}} z}\right], \\
& D_{c, \bar{c}}^{\pi^{+}} \quad\left(z, m_{c}^{2}\right)=N_{c}^{\pi^{+}} z^{\alpha_{c}^{\pi^{+}}}(1-z)^{\beta_{c}^{\pi^{+}}}\left[1-e^{-\gamma_{c}^{\pi^{+}}} z\right], \\
& D_{b, \bar{b}}^{\pi^{+}} \quad\left(z, m_{b}^{2}\right)=N_{b}^{\pi^{+}} z^{\alpha_{b}^{\pi^{+}}}(1-z)^{\beta_{b}^{\pi^{+}}}\left[1-e^{-\gamma_{b}^{\pi^{+}} z}\right] .
\end{aligned}
$$

Considering the constituent quark composition of kaon $\left|K^{+}\right\rangle=|u \bar{s}\rangle$, we define kaon functional form for light partons as following

$$
\begin{array}{cl}
D_{u}^{K^{+}} \quad\left(z, \mu_{0}^{2}\right)=N_{u}^{K^{+}} z^{\alpha_{u}^{K^{+}}}(1-z)^{\beta_{u}^{K^{+}}}\left[1-e^{-\gamma_{u}^{K^{+}} z}\right], \\
D_{\bar{s}}^{K^{+}} \quad\left(z, \mu_{0}^{2}\right)=N_{\bar{s}}^{K^{+}} z^{\alpha_{\bar{s}}^{K^{+}}}(1-z)^{\beta_{\bar{s}}^{K^{+}}}\left[1-e^{-\gamma_{\bar{s}}^{K^{+}} z}\right], \\
D_{d, \bar{d}, \bar{u}, s}^{K^{+}} \quad\left(z, \mu_{0}^{2}\right)=N_{d}^{K^{+}} z^{\alpha_{d}^{K^{+}}}(1-z)^{\beta_{d}^{K^{+}}}\left[1-e^{-\gamma_{d}^{K^{+}} z}\right] .
\end{array}
$$

We apply a new form of kaon FF for the strange-quark because of its mass against the $u$-quark mass. Kaon fragmentation functions for sea quarks defined by considering isospin symmetry between them. Gluon and heavy quarks FFs are defined as following

$$
\begin{aligned}
& D_{g}^{K^{+}}\left(z, \mu_{0}^{2}\right)=N_{g}^{K^{+}} z^{\alpha_{g}^{K^{+}}}(1-z)^{\beta_{g}^{K^{+}}}\left[1-e^{-\gamma_{g}^{K^{+}}} z\right], \\
& D_{c, \bar{c}}^{K^{+}}\left(z, m_{c}^{2}\right)=N_{c}^{K^{+}} z^{\alpha_{c}^{K^{+}}}(1-z)^{\beta_{c}^{K^{+}}} \\
& D_{b, \bar{b}}^{K^{+}}\left(z, m_{b}^{2}\right)=N_{b}^{K^{+}} z^{\alpha_{b}^{K^{+}}}(1-z)^{\beta_{b}^{K^{+}}} .
\end{aligned}
$$

In our analysis, the heavy partons parameters $\gamma_{c}$ and $\gamma_{b}$ impress the $\chi^{2}$ value. We use Eq. 13 as a functional form for all partons of pion and our decision to put or omit the term $\left[1-e^{-\gamma_{i} z}\right]$ for different flavors of kaon FFs is based on getting the best $\chi^{2}$. In reported parameters in Refs. [22 24] some parameters are fixed or in the other word the simple parametrization form is used. In Ref. 23] one of the gluon parameters is fixed for pion and kaon and DSS model 24] uses the simple parametrization for $c$ and $b$-quarks for $\pi^{+}$and $K^{+}$mesons.

According to the parton structure of $\pi^{-}(\bar{u} d)$ and $K^{-}(\bar{u} s)$, parton fragmentation functions could be calculated for $\pi^{-}$and $K^{-}$as

$$
\begin{aligned}
D_{i}^{\pi^{-}}\left(z, \mu_{0}^{2}\right) & =D_{\bar{i}}^{\pi^{+}}\left(z, \mu_{0}^{2}\right), \\
D_{i}^{K^{-}}\left(z, \mu_{0}^{2}\right) & =D_{\bar{i}}^{K^{+}}\left(z, \mu_{0}^{2}\right),
\end{aligned}
$$

where $i=u, d, s, c, b$ and also for gluon fragmentation functions

$$
\begin{aligned}
D_{g}^{\pi^{-}}\left(z, \mu_{0}^{2}\right) & =D_{g}^{\pi^{+}}\left(z, \mu_{0}^{2}\right), \\
D_{g}^{K^{-}}\left(z, \mu_{0}^{2}\right) & =D_{g}^{K^{+}}\left(z, \mu_{0}^{2}\right) .
\end{aligned}
$$

\section{QCD analysis of Experimental data and global minimization of $\chi^{2}$}

The free parameters in the functional forms of $\pi^{+}$and $K^{+}$FFs ( Eqs. (15) 18) ) are determined by minimizing $\chi^{2}$ for differential cross section and asymmetry experimental data (i.e. $\left(1 / \sigma_{\text {tot }} \cdot d \sigma / d z\right)_{\text {exp }}$ and $\left.\left(A_{1}^{N, H}\right)_{\text {exp }}\right)$ in $x$ space. The global $\chi^{2}$ is defined as

$$
\chi_{g l o b a l}^{2}=\sum_{n} w_{n} \chi_{n}^{2}
$$

where $n$ is the number of experimental data group and $w_{n}$ denotes a weight factor for the $n$-th experimental data group. The $\chi_{n}^{2}$ is defined as

$$
\chi_{n}^{2}=\left(\frac{1-N_{n}}{\Delta N_{n}}\right)^{2}+\sum_{j=1}^{k}\left(\frac{N_{n} E_{j}-T_{j}}{N_{n} \sigma_{j}^{E}}\right)^{2},
$$

where $T_{j}$ and $E_{j}$ are the theoretical and experimental values of $1 / \sigma_{\text {tot }} \cdot d \sigma / d z$ for $e^{+} e^{-}$SIA data and $A_{1}^{N, H}$ for SIDIS data and $\sigma_{j}^{E}$ is the error of corresponding experimental value. Here, the summation goes over the $k$ bins of the experimental data. $\Delta N_{n}$ related to the experimental normalization uncertainty which is reported by the experiments and $N_{n}$ corresponds to an overall normalization factor which is refer to the data of experiments. Usually $N_{n}$ is gotten from first minimization and we fix it in the second minimization. In our global fit we take SIA experimental data from LEP (ALEPH, DELPHI and OPAL collaborations), SLAC (BaBar, SLD and TPC collaborations), DESY (TASSO collaboration) 
TABLE I: Fit parameters for the parton FFs into the charged pion $\left(\pi^{+}\right)$at LO, $D_{i}^{\pi^{+}}\left(z, \mu_{0}\right)$. The starting scale is taken to be $\mu_{0}^{2}=1 \mathrm{GeV}^{2}$ for light partons and gluon and also $\mu_{0}^{2}=m_{c}^{2}$ and $\mu_{0}^{2}=m_{b}^{2}$ for $c$ and $b$-quarks.

\begin{tabular}{ccccc}
\hline \hline flavor $i$ & $N_{i}$ & $\alpha_{i}$ & $\beta_{i}$ & $\gamma_{i}$ \\
\hline$u, \bar{d}$ & $0.841 \pm 0.435$ & $-2.041 \pm 0.417$ & $0.803 \pm 0.284$ & $1.170 \pm 0.789$ \\
$\bar{u}, d, s, \bar{s}$ & $2.509 \pm 1.143$ & $-1.514 \pm 0.269$ & $4.610 \pm 1.083$ & $2.123 \pm 1.189$ \\
$c, \bar{c}$ & $1.059 \pm 0.788$ & $-1.918 \pm 0.364$ & $3.325 \pm 1.060$ & $1.697 \pm 1.447$ \\
$b, \bar{b}$ & $1.062 \pm 0.596$ & $-2.043 \pm 0.218$ & $5.902 \pm 1.328$ & $1.750 \pm 1.044$ \\
$g$ & $59.993 \pm 10.523$ & $0.939 \pm 1.961$ & $5.801 \pm 5.394$ & $1.219 \pm 3.508$ \\
\hline \hline
\end{tabular}

TABLE II: Values of fit parameters for the $\pi^{+}$meson at NLO in the starting scale.

\begin{tabular}{ccccc}
\hline \hline flavor $i$ & $N_{i}$ & $\alpha_{i}$ & $\beta_{i}$ & $\gamma_{i}$ \\
\hline$u, \bar{d}$ & $1.049 \pm 0.563$ & $-1.916 \pm 0.421$ & $0.977 \pm 0.304$ & $0.964 \pm 0.65$ \\
$\bar{u}, d, s, \bar{s}$ & $9.968 \pm 7.441$ & $-0.516 \pm 0.481$ & $5.952 \pm 1.565$ & $1.898 \pm 1.885$ \\
$c, \bar{c}$ & $0.946 \pm 0.859$ & $-1.723 \pm 0.451$ & $3.590 \pm 1.280$ & $1.947 \pm 1.981$ \\
$b, \bar{b}$ & $0.869 \pm 0.550$ & $-2.059 \pm 0.234$ & $5.803 \pm 1.460$ & $1.561 \pm 1.054$ \\
$g$ & $219.507 \pm 44.789$ & $1.073 \pm 0.362$ & $7.505 \pm 1.140$ & $2.142 \pm 1.411$ \\
\hline \hline
\end{tabular}

and KEK (Belle and TOPAZ collaborations). The energy scales of experimental data are from $10.52 \mathrm{GeV}$ to $91.2 \mathrm{GeV}$ [8 17]. In the reported data without discrimination of hadron species, authors distinguished between four cases; fragmentation of $u, d, s$-quarks, $c$-quark only, $b$-quark, and all five quark flavors $(u, d, s, c$, and $b)$. These categories are just in DELPHI and SLD data 9, 10, 12. Also the BaBar and Belle collaborations [16, 17] reported inclusive hadron production cross sections recently at a center-of-mass energy of $10.54 \mathrm{GeV}$ and $10.52 \mathrm{GeV}$, respectively. Since the center-of-mass energies are below the threshold to produce a $b \bar{b}$ pair, these data containing a pure $e^{+} e^{-} \rightarrow q \bar{q}$ sample, where $q=u, d, s, c$. Although most of the precision $e^{+} e^{-}$annihilation data, are limited to results from experiments at LEP and SLAC at the energy scale of the $M_{Z}$, the large data samples are available at BaBar and Belle collaborations at $Q=10.54 \mathrm{GeV}$ and $Q=10.52 \mathrm{GeV}$, respectively. In addition, these two collaborations reported the differential cross sections at $z>0.7$.

We also provide SIDIS experimental data for $A_{1}^{p, \pi^{+}}$, $A_{1}^{p, \pi^{-}}, A_{1}^{d, \pi^{+}}, A_{1}^{d, \pi^{-}}, A_{1}^{p, K^{+}}, A_{1}^{p, K^{-}}, A_{1}^{d, K^{+}}$and $A_{1}^{d, K^{-}}$ from HERMES05 [25], COMPASS09 26] and COMPASS10 [27]. The energy scales of SIDIS experimental data are from $1.16 \mathrm{GeV}^{2}$ to $55.60 \mathrm{GeV}^{2}$.

In each collaboration small $z$ data are excluded since the splitting functions in evolution equations lead to negative FFs in their NLO part for $z \ll 1$, additionally mass corrections are more important in this region. So we exclude regions where mass corrections and the singular small $z$ behavior of the splitting functions are effective. The $z>0.1$ is used for the data which have $\sqrt{s}<M_{Z}$ and the $z>0.05$ is used for data which have $\sqrt{s}=M_{Z}$.

\section{Neighborhood of global minimum and Hessian method}

In recent years, the assessment of uncertainties is significant progress in QCD analysis of PDFs and FFs 65 67) and among different approaches Lagrange Multiplier (LM) technique and Hessian method are the most reliable ones. While LM technique avoids any approximations or assumptions about the behavior of the $\chi^{2}$ on the parameters, the only drawback to this method is that its calculation is slow because it needs a separate minimizations for each parameters.

Since we use Hessian or error matrix approach in our analysis, the outline of this method is explained. The basic assumption of Hessian approach is a quadratic expansion of the $\chi^{2}$ in the fit parameters $a_{i}$ near the global minimum

$$
\Delta \chi^{2} \equiv \chi^{2}-\chi_{\min }^{2}=\sum_{i, j=1}^{n} H_{i j}\left(a_{i}-a_{i}^{0}\right)\left(a_{j}-a_{j}^{0}\right),
$$

with

$$
H_{i j}=\left.\frac{1}{2} \frac{\partial^{2} \chi^{2}}{\partial a_{i} \partial a_{j}}\right|_{\min },
$$

where $H_{i j}$ are the elements of the Hessian matrix. Since Hessian matrix and its inverse $\left(C \equiv H^{-1}\right)$, which is the error matrix, are symmetric, they have a set of $n$ orthogonal eigenvectors $v_{i k}$ with eigenvalues $\lambda_{k}$

$$
\begin{aligned}
\sum_{j=1}^{n} C_{i j} v_{j k} & =\lambda_{k} v_{i k} \\
\sum_{i=1}^{n} v_{i j} v_{i k} & =\delta_{j k} .
\end{aligned}
$$


TABLE III: Fit parameters for the parton FFs into the charged kaon $\left(K^{+}\right)$at LO $\left(D_{i}^{K^{+}}\left(z, \mu_{0}\right)\right)$ in the starting scale.

\begin{tabular}{ccccc}
\hline \hline flavor $i$ & $N_{i}$ & $\alpha_{i}$ & $\beta_{i}$ & $\gamma_{i}$ \\
\hline$u$ & $4.415 \pm 5.203$ & $-0.388 \pm 0.315$ & $1.486 \pm 0.725$ & $0.412 \pm 0.513$ \\
$\bar{s}$ & $29.284 \pm 15.300$ & $1.395 \pm 1.865$ & $2.524 \pm 1.293$ & $0.848 \pm 2.413$ \\
$\bar{u}, d, \bar{d}, s$ & $6.231 \pm 3.877$ & $-0.398 \pm 0.106$ & $6.273 \pm 2.337$ & $2.435 \pm 2.168$ \\
$c, \bar{c}$ & $4.853 \pm 3.343$ & $0.245 \pm 0.411$ & $4.530 \pm 1.090$ & - \\
$b, \bar{b}$ & $8.324 \pm 2.947$ & $0.076 \pm 0.084$ & $8.841 \pm 1.836$ & - \\
$g$ & $1.309 \pm 3.529$ & $8.871 \pm 4.530$ & $0.293 \pm 2.44$ & $0.165 \pm 2.732$ \\
\hline \hline
\end{tabular}

TABLE IV: Values of fit parameters for the $K^{+}$meson at NLO in the starting scale.

\begin{tabular}{ccccc}
\hline \hline flavor $i$ & $N_{i}$ & $\alpha_{i}$ & $\beta_{i}$ & $\gamma_{i}$ \\
\hline$u$ & $0.660 \pm 0.156$ & $-1.584 \pm 0.342$ & $0.858 \pm 0.227$ & $0.390 \pm 0.107$ \\
$\bar{s}$ & $17.769 \pm 7.775$ & $0.708 \pm 0.390$ & $2.479 \pm 0.316$ & $0.665 \pm 0.218$ \\
$\bar{u}, d, \bar{d}, s$ & $6.467 \pm 1.587$ & $0.028 \pm 0.547$ & $7.338 \pm 0.819$ & $3.299 \pm 1.282$ \\
$c, \bar{c}$ & $7.217 \pm 1.013$ & $0.550 \pm 0.113$ & $5.366 \pm 0.314$ & - \\
$b, \bar{b}$ & $14.675 \pm 3.227$ & $0.293 \pm 0.080$ & $10.882 \pm 0.943$ & - \\
$g$ & $2.383 \pm 0.381$ & $5.714 \pm 0.696$ & $0.892 \pm 0.085$ & $53542.030 \pm 5.859$ \\
\hline \hline
\end{tabular}

The parameter variation around the global minimum can be expanded in a basis of eigenvectors and eigenvalues, that is,

$$
a_{i}-a_{i}^{0}=\sum_{k=1}^{n} e_{i k} z_{k}
$$

where $e_{i k} \equiv \sqrt{\lambda_{k}} v_{i k}$. Using Eqs. 26] and 27 it can be shown that the expansion of the $\chi^{2}$ in the fit parameters $a_{i}$ near the global minimum Eq. 23 reduces to

$$
\chi^{2}=\chi_{\min }^{2}+\sum_{k=1}^{n} z_{k}^{2}
$$

where $\sum_{k=1}^{n} z_{k}^{2} \leq T^{2}$ is the interior of a sphere of radius $T$. The eigenvector sets $S_{k}^{ \pm}$are defined by choosing $T=\left(\Delta \chi^{2}\right)^{1 / 2}$ and corresponding positive and negative of eigenvector directions are defined as following

$$
z_{i}\left(S_{k}^{ \pm}\right)= \pm T \delta_{i k}
$$

Using the last equation, the $a_{i}$ parameters that specify the eigenvector basis sets $S_{k}^{ \pm}$at a fixed value of $\alpha_{S}$, are given by

$$
a_{i}\left(S_{k}^{ \pm}\right)=a_{i}^{0} \pm T e_{i k} .
$$

In the standard parameter-fitting criterion, the errors are given by the choice of tolerance $T=\Delta \chi^{2}=1$. Also we can determine the size of uncertainties applying Hessian method based on correspondence between the confidence level $P$ and $\Delta \chi^{2}$ with the number of fitting parameters $N$

$$
P=\int_{0}^{\Delta \chi^{2}} \frac{1}{2 \Gamma(N / 2)}\left(\frac{x}{2}\right)^{\frac{N}{2}-1} \exp \left(-\frac{x}{2}\right) d x
$$

where we get $P=0.68$ as the confidence level and $\Delta \chi^{2}=$ 22.43 and $\Delta \chi^{2}=24.58$ are obtained for pion and kaon, respectively.

The uncertainty on a quantity $F\left(a_{i}\right)$ which is attributive function of the input parameters obtained in the QCD fit procedure at the scale $Q_{0}$, is obtained applying the simple Hessian method

$$
\Delta F=T \sqrt{\sum_{i, j=1}^{n} \frac{\partial F}{\partial a_{i}} C_{i j} \frac{\partial F}{\partial a_{j}}} .
$$

\section{E. The method of error calculation}

According to Eqs. (15, 18) the evolved fragmentation functions for pion and kaon are attributive functions of the input parameters which are calculated from the fit. Their standard linear errors are given by Gaussian error propagation. If $D_{i}^{H}\left(z ; Q^{2}\right)$ is the evolved fragmentation density at $Q^{2}$ then Gaussian error propagation is defined as

$$
\left[\delta D_{i}^{H}(z)\right]^{2}=\Delta \chi^{2} \sum_{j, k}^{n} \frac{\partial D_{i}^{H}\left(z, a_{j}\right)}{\partial a_{j}}\left(H_{j k}\right)^{-1} \frac{\partial D_{i}^{H}\left(z, a_{k}\right)}{\partial a_{k}}
$$

where $\Delta \chi^{2}$ is the allowed variation in $\chi^{2}$ and $\left.a_{j}\right|_{j=1} ^{n}$ are free parameters and $n$ is the number of parameters in the global fit. Also $H_{j k}$ is Hessian or covariance matrix of the parameters determined in the QCD analysis at the initial scale $Q_{0}^{2}$ and it is defined in Eq. 24. Consequently we can calculate the uncertainties of any FFs by using Hessian or covariance matrix based on the 
TABLE V: The individual $\chi^{2}$ values and the fitted normalization in the LO for each collaboration and the total $\chi^{2}$ fit for $\pi^{+}$.

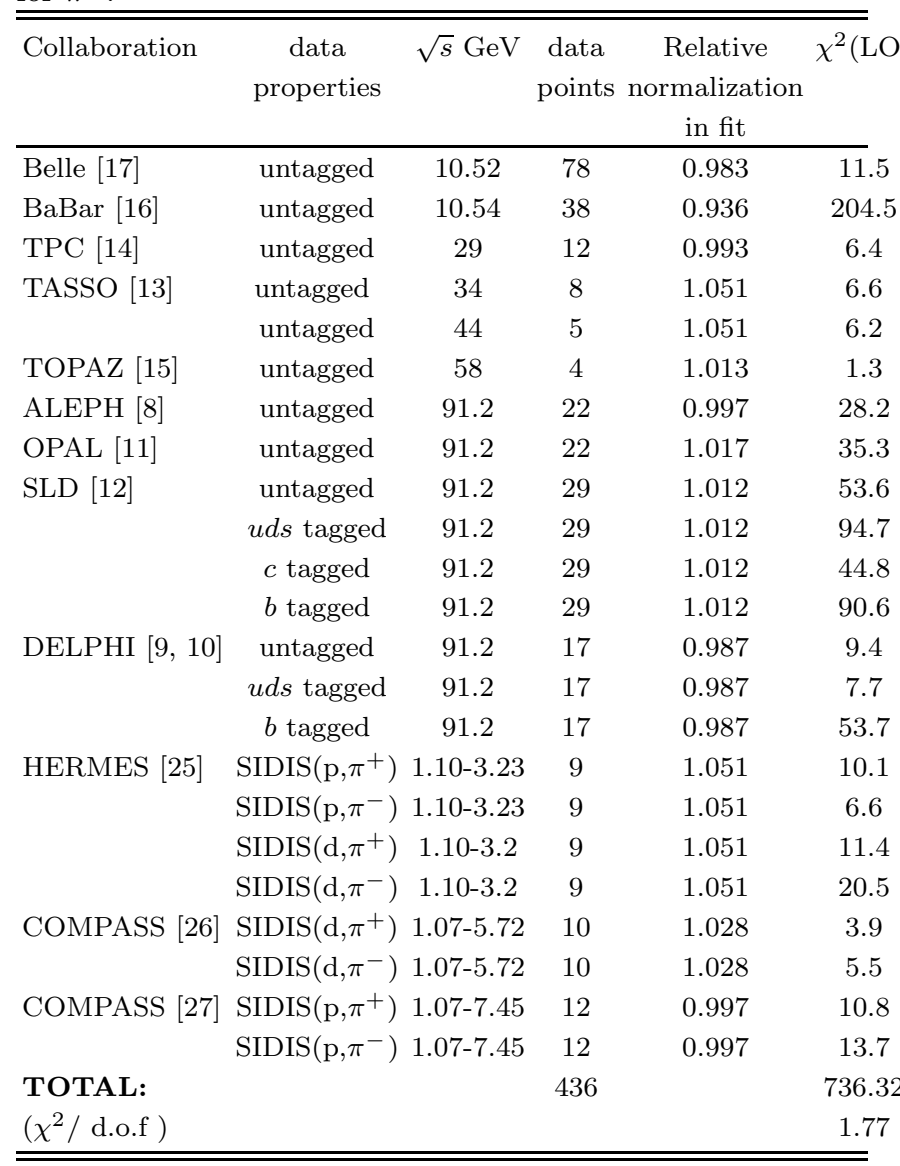

Gaussian method at any value of $Q^{2}$ by the QCD evolution. More information and detailed discussion can be found in Refs. 23, 68, 69].

\section{INCLUSIVE $\pi^{+} / K^{+}$-MESONS PRODUCTION IN TOP-QUARK DECAY}

Nowadays, the CERN Large Hadron Collider (LHC) is a superlative machine to produce top-quark pairs so that at design energy $\sqrt{S}=14 \mathrm{TeV}$ and design luminosity $\mathcal{L}=10^{34} \mathrm{~cm}^{-2} \mathrm{~s}^{-1}$ in each of the four experiments it is expected to produce about 90 million top-quark pairs per year [70]. This much volume of events will allow us to determine the properties of the top-quark, such as its mass $m_{t}$, branching fractions and matrix elements $V_{t q}$ of the Cabibbo-Kobayashi-Maskawa (CKM) 71] with high precision. Because of its large mass, the top-quark decays so rapidly so that it has no time to hadronize and due to $\left|V_{t b}\right| \approx 1$, top-quarks almost decay to bottom- quarks, via the decay mode $t \rightarrow b W^{+}$in the standard model (SM). Bottom-quarks also hadronize, via $b \rightarrow H+X$, before they decay, and thus the decay mode $t \rightarrow H W^{+}+X$ is of prime importance where $\mathrm{H}$ refers to the detected out- going hadron. A particular interest at LHC is to study the scaled energy distributions of outgoing hadron.

In this section, we study the energy spectrum of the inclusive light mesons including $\pi^{+}$and $K^{+}$in top-quark decay working in the zero-mass variable-flavor-number scheme(ZM-VFNs).

We wish to study the inclusive production of a light meson in the decay process

$$
t \rightarrow b+W^{+}(g) \rightarrow \pi^{ \pm} / K^{ \pm}+X,
$$

where $X$ stands for the unobserved final state. The gluon in Eq. (34) contributes to the real radiation at NLO and both the $b$-quark and the gluon may hadronize to the outgoing light mesons. To obtain the energy distribution of light hadrons we use the realistic FFs obtained in our approach.

To study the energy spectrum of outgoing meson it would be convenient to introduce the scaled energy fractions $x_{i}=E_{i} / E_{b}^{\max }(i=b, g, H)$ where $H$ stands for the light mesons. In the top-qurak rest frame, the energies range $0 \leq\left(E_{b}, E_{g}\right) \leq\left(m_{t}^{2}-m_{W}^{2}\right) /\left(2 m_{t}\right)$ and $m_{H} \leq E_{H} \leq\left(m_{t}^{2}+m_{H}^{2}-m_{W}^{2}\right) /\left(2 m_{t}\right)[72]$.

We wish to calculate the partial decay width of process (34) differential in $x_{H}\left(d \Gamma / d x_{H}\right)$ at NLO in the

TABLE VI: The individual $\chi^{2}$ values and the fitted normalization in the NLO for each collaboration and the total $\chi^{2}$ fit for $\pi^{+}$

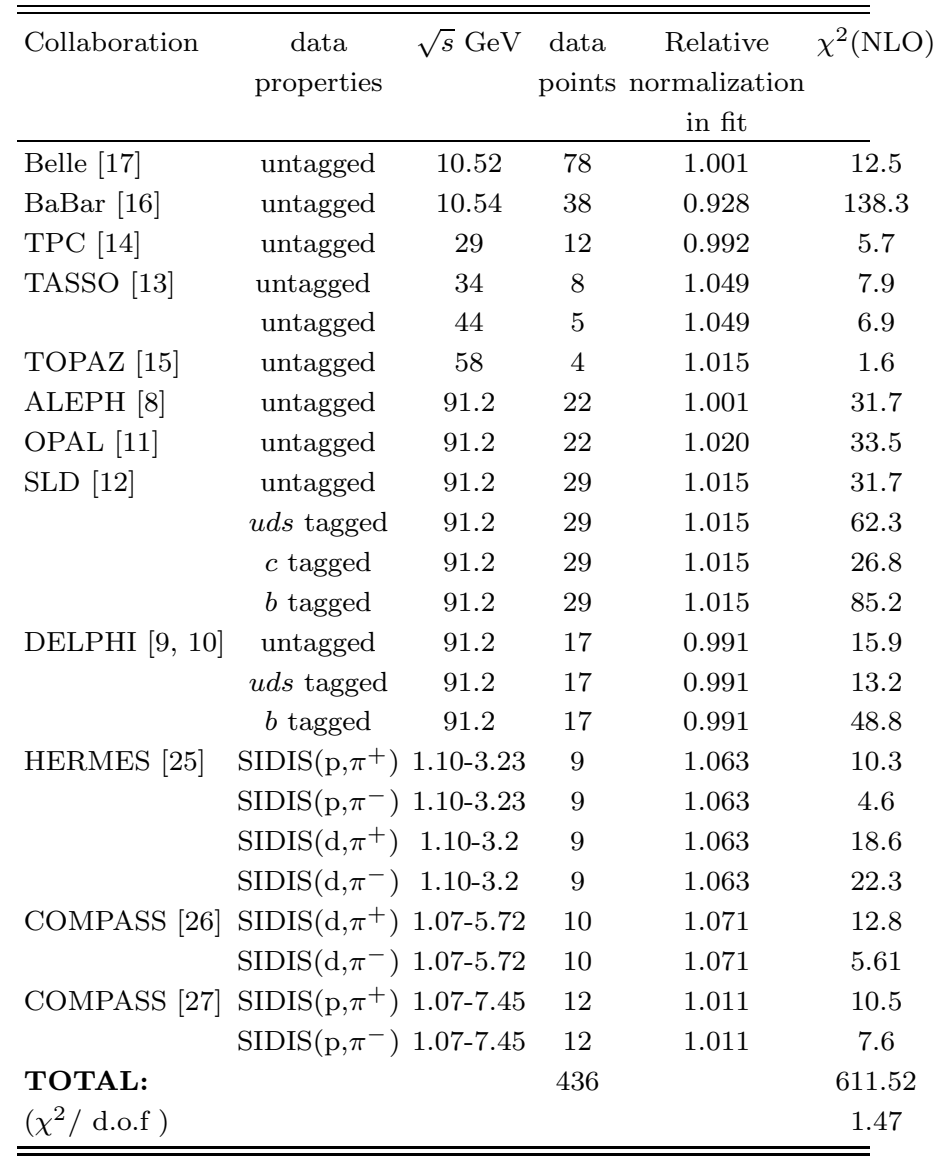


ZM-VFN scheme. Considering the factorization theorem of the QCD [30], the energy distribution of a hadron $\mathrm{H}$ can be expressed as the convolution of the parton-level spectrum with the fragmentation densities $D_{i}^{H}\left(z, \mu_{F}\right)$, describing the hadronization $i \rightarrow H$,

$$
\frac{d \Gamma}{d x_{H}}=\sum_{i=b, g} \int_{x_{i}^{\min }}^{x_{i}^{\max }} \frac{d x_{i}}{x_{i}} \frac{d \hat{\Gamma}_{i}}{d x_{i}}\left(\mu_{R}, \mu_{F}\right) D_{i}^{H}\left(\frac{x_{H}}{x_{i}}, \mu_{F}\right)
$$

where $d \hat{\Gamma}_{i} / d x_{i}$ is the parton-level differential width of the process $t \rightarrow i+W^{+}(i=b, g)$, which are being extracted from Ref. [72]. Here, $\mu_{F}$ and $\mu_{R}$ are the factorization and the renormalization scales which are set to $\mu_{R}=\mu_{F}=m_{t}$. The values of all $\mathrm{FF}$ parameters are listed in Tables II II, III and IV. Since these FFs are parameterized at the low factorization scale, extraction of the FFs at each arbitrary scale of energy should be performed using the grids and FORTRAN routines based on solving DGLAP equations.

\section{FIT RESULTS}

Now we are in a situation to explain our global analysis of fragmentation functions for pions and kaons results. We compare our calculated cross section and double spin asymmetry results with the experimental data and find a good agreement.

According to last section that we introduce the experimental data for our fits, we present our results of the optimum fits for the fragmentation parameters of the $\pi^{+}$- and $K^{+}$-mesons in the initial scale $\mu_{0}$ at LO and NLO. Also $\chi^{2}$ and normalization fit values for each individual collaboration are reported. On the other hand we are interested in presenting how much the asymmetry SIDIS data effect for determination of pion and kaon FFs. Also the FFs comparisons are made with the results obtained in the other FFs analysis in Refs. [23, 24, 28]. We also briefly present the dependance of $\Delta \chi^{2}$ global along some random samples of eigenvector directions to illustrate the deviations of the $\Delta \chi^{2}$ function from the expected quadratic dependence. At the end we show our prediction for energy spectrum of pion and kaon as light mesons in top decay.

\section{A. Experimental data and our analysis}

Experimental data for inclusive hadron production in SIA cover orders of energy magnitude from $10.52 \mathrm{GeV}$ to the mass of the $Z$-boson. By adding SIDIS experimental data the range of energy is extended and cover low energy from $1.16 \mathrm{GeV}^{2}$ to $55.60 \mathrm{GeV}^{2}$. In Figs. 1 and 2 , using new functional form of FFs we compare our results for $\frac{1}{\sigma_{t o t}} \frac{d \sigma^{i}}{d z}(i=\pi$ or $K)$ with the data at $\mu^{2}=M_{Z}^{2}$ reported by ALEPH, DELPHI, OPAL and SLD collaborations at NLO. In these figures we separate the light, charm and
TABLE VII: The individual $\chi^{2}$ values and the fitted normalization in the LO for each collaboration and the total $\chi^{2}$ fit for $K^{+}$.

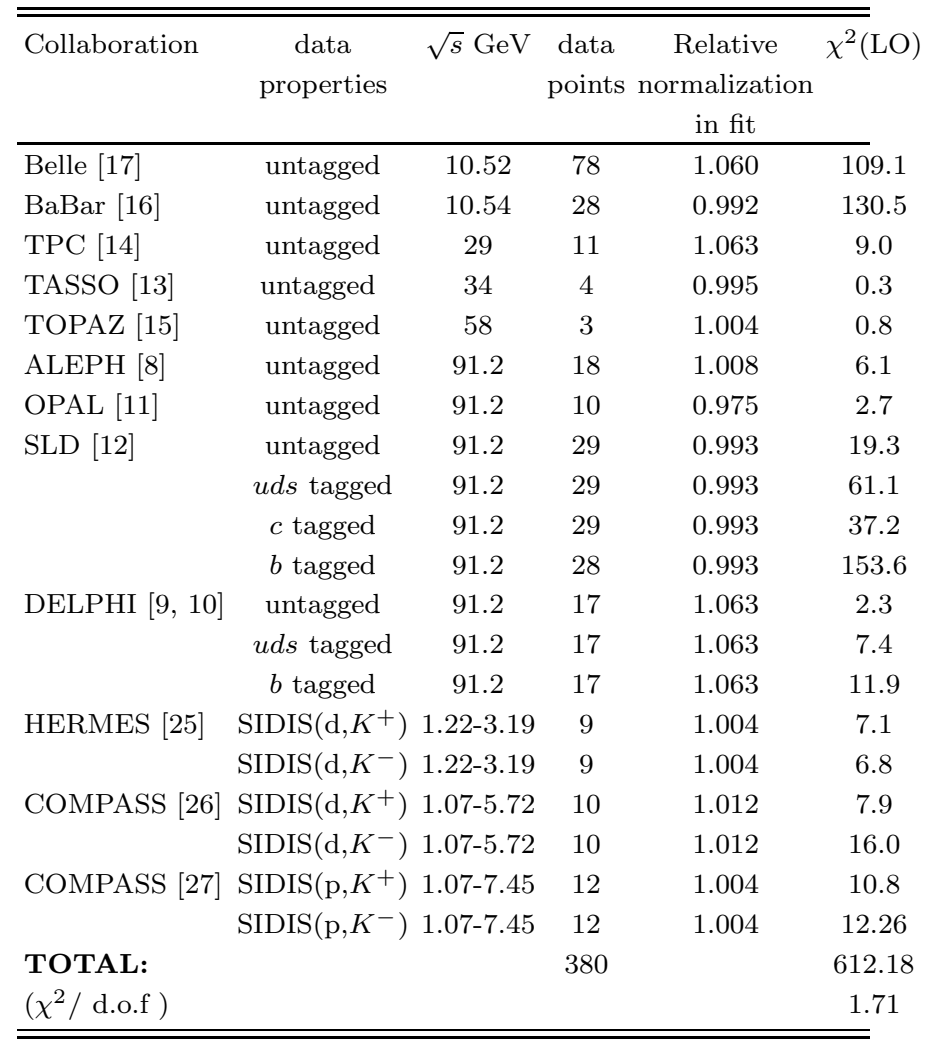

bottom tagged cross sections and most of the diagrams show a remarkable agreement between our results and experimental data.

Comparing other FFs models in these figures also gives a nice altogether description of our model with the AKK set [28] that included hadron production data in electronpositron and hadron-hadron scattering data, the DSS set 24] that included electron-positron, lepton-nucleon and hadron-hadron scattering data and HKNS set 23] that included electron-positron data. However, as Fig. 1 shows, due to the largeness of $\chi^{2}$ contributions for SLD and DELPHI $b$ tagged (see Table $\mathrm{V}$ and $\mathrm{VI}$ ) some points are outside of the curves. Generally, $\chi^{2}$ values of the heavy flavors, in particular, $b$ tagged data are larger than the other data (see Tables V. VI, VII and VIII) that it might be caused by some extent to contaminations from weak decay.

Recent differential cross section data from Belle and BaBar collaborations are included in our analysis. In Fig. 3] our results for pion and kaon at $Q=10.52 \mathrm{GeV}$ and $Q=10.54 \mathrm{GeV}$ are compered with these data. Also the other FFs models are compared with Belle and BaBar data and this figure shows a nice agreement between our model and these data.

Figs. 4]and 5]present the extracted values of $A_{1}$ for proton and deuteron from the global fit for $\pi^{+}, \pi^{-}, K^{+}$and $K^{-}$ at NLO in comparison with the SIDIS data from HER- 

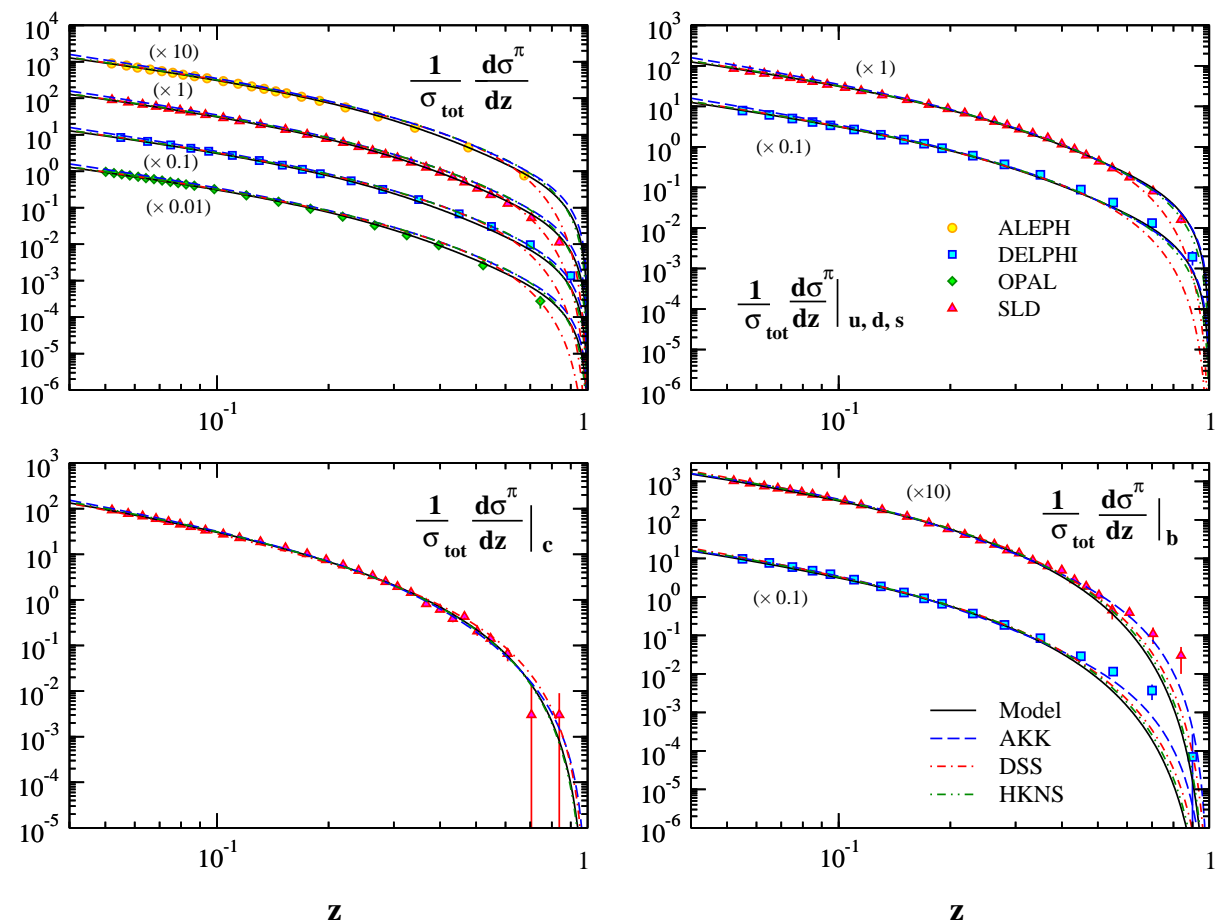

FIG. 1: Comparison of our NLO results for $\frac{1}{\sigma_{t o t}} \frac{d \sigma^{\pi}}{d z}$ in total and tagged cross sections with pion production data at $Q^{2}=M_{Z}^{2}$ by ALEPH, DELPHI, OPAL and SLD [8 12]. Our model, is denoted Model, also is compered with the other models [23, 24, 28].
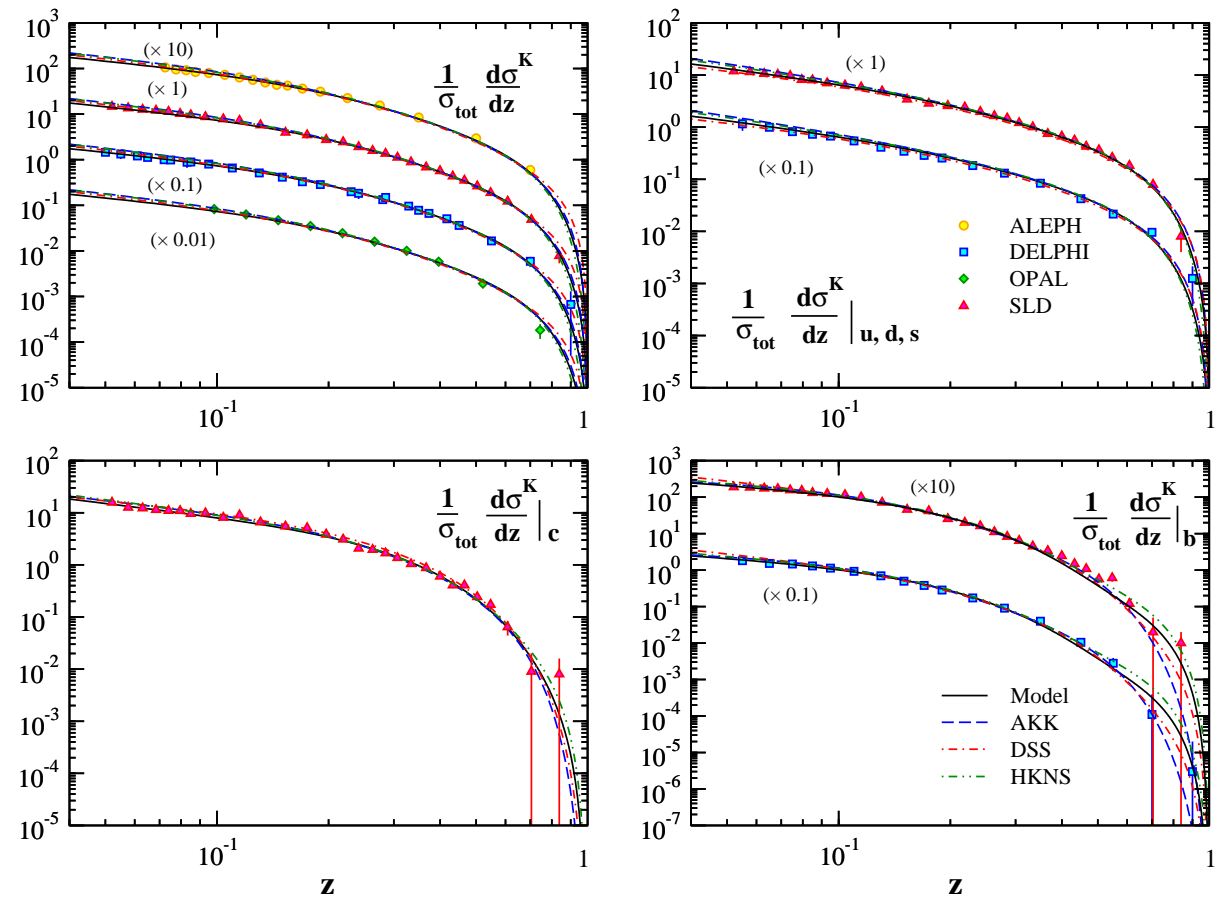

FIG. 2: Comparison of our NLO results for $\frac{1}{\sigma t o t} \frac{d \sigma^{K}}{d z}$ in total and tagged cross sections with kaon production data at $Q^{2}=M_{Z}^{2}$ by ALEPH, DELPHI, OPAL and SLD [8 12]. Our model, is denoted Model, also is compered with the other models [23, 24, 28]. 

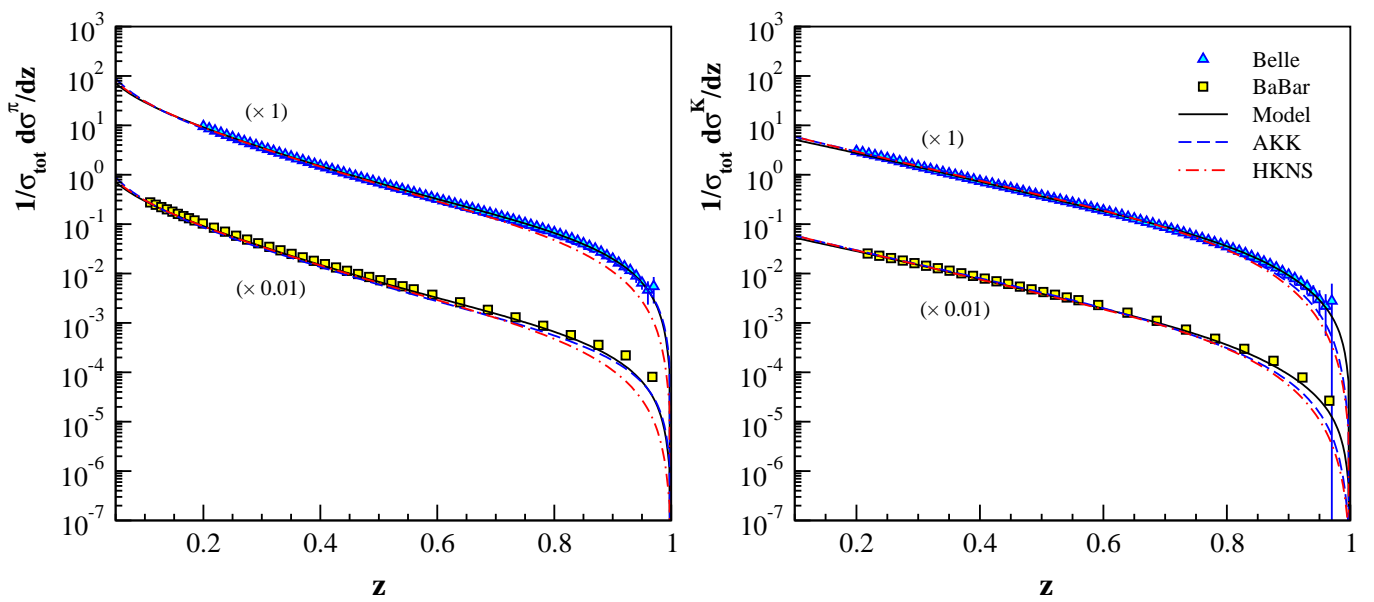

FIG. 3: Comparison of our NLO results for $\frac{1}{\sigma_{t o t}} \frac{d \sigma^{i}}{d z},(i=\pi, K)$ in total cross sections with pion and kaon production data at $Q=10.54 \mathrm{GeV}$ by BaBar [16] and $Q=10.52 \mathrm{GeV}$ by Belle [17]. Our model, is denoted Model, also is compered with the other models $[23,28]$.

MES and COMPASS 25 27]. The extraction of double spin asymmetry data from the global fit for fragmenta-

TABLE VIII: The individual $\chi^{2}$ values and the fitted normalization in the NLO for each collaboration and the total $\chi^{2}$ fit for $K^{+}$.

\begin{tabular}{|c|c|c|c|c|c|}
\hline \multirow[t]{2}{*}{ Collaboration } & \multirow{2}{*}{$\begin{array}{c}\text { data } \\
\text { properties }\end{array}$} & \multirow[t]{2}{*}{$\sqrt{s} \mathrm{GeV}$} & \multirow{2}{*}{\multicolumn{2}{|c|}{$\begin{array}{cc}\text { data } & \text { Relative } \\
\text { points normalization } \\
\text { in fit }\end{array}$}} & \multirow{2}{*}{$\chi^{2}(\mathrm{NLO}$} \\
\hline & & & & & \\
\hline Belle [17] & untagged & 10.52 & 78 & 1.029 & $\overline{10} 5.7$ \\
\hline BaBar [16] & untagged & 10.54 & 28 & 0.974 & 100.8 \\
\hline TPC [14] & untagged & 29 & 11 & 1.041 & 7.6 \\
\hline TASSO [13] & untagged & 34 & 4 & 0.992 & 0.3 \\
\hline TOPAZ [15] & untagged & 58 & 3 & 1.004 & 0.8 \\
\hline ALEPH [8] & untagged & 91.2 & 18 & 1.017 & 4.9 \\
\hline OPAL [11] & untagged & 91.2 & 10 & 0.983 & 2.8 \\
\hline \multirow[t]{4}{*}{ SLD [12] } & untagged & 91.2 & 29 & 1.003 & 19.1 \\
\hline & $u d s$ tagged & 91.2 & 29 & 1.003 & 67.6 \\
\hline & $c$ tagged & 91.2 & 29 & 1.003 & 42.7 \\
\hline & $b$ tagged & 91.2 & 28 & 1.003 & 116.5 \\
\hline \multirow[t]{3}{*}{ DELPHI $[9,10]$} & untagged & 91.2 & 17 & 1.084 & 2.6 \\
\hline & $u d s$ tagged & 91.2 & 17 & 1.084 & 6.9 \\
\hline & $b$ tagged & 91.2 & 17 & 1.084 & 11.8 \\
\hline \multirow[t]{2}{*}{ HERMES [25] } & $\operatorname{SIDIS}\left(\mathrm{d}, K^{+}\right)$ & $1.22-3.19$ & 9 & 1.009 & 6.0 \\
\hline & $\operatorname{SIDIS}\left(\mathrm{d}, K^{-}\right)$ & $1.22-3.19$ & 9 & 1.009 & 8.1 \\
\hline \multirow[t]{2}{*}{ COMPASS [26] } & $\operatorname{SIDIS}\left(\mathrm{d}, K^{+}\right)$ & $1.07-5.72$ & 10 & 1.032 & 7.2 \\
\hline & $\operatorname{SIDIS}\left(\mathrm{d}, K^{-}\right)$ & $1.07-5.72$ & 10 & 1.032 & 20.4 \\
\hline \multirow[t]{2}{*}{ COMPASS [27] } & $\operatorname{SIDIS}\left(\mathrm{p}, K^{+}\right)$ & $1.07-7.45$ & 12 & 1.013 & 6.5 \\
\hline & $\operatorname{SIDIS}\left(\mathrm{p}, K^{-}\right)$ & $1.07-7.45$ & 12 & 1.013 & 15.3 \\
\hline TOTAL: & & & 380 & & 551.32 \\
\hline$\left(\chi^{2} /\right.$ d.o.f $)$ & & & & & 1.54 \\
\hline
\end{tabular}

tion functions is done for the first time and as it is shown the overall agreement of the experimental data sets in the global analysis is great. Some of the theoretical analysis such as Refs. 3, 43] use the asymmetry data from DIS and SIDIS to calculate the polarized parton distributions.

\section{B. Fit results for $\pi^{+}$and $K^{+}$FFs}

According to the scenarios defined for the fragmentation functions of $\pi^{+}$and $K^{+}$-mesons at the starting scales, 20 and 22 parameters have to be determined, respectively. These parameters are listed in Tables I and II for $\pi^{+}$and in Tables III and IV for $K^{+}$at LO and NLO. The initial scales for the $b \rightarrow \pi^{+} / K^{+}$and $c \rightarrow \pi^{+} / K^{+}$FFs are $\mu_{0}^{2}=m_{b}^{2}$ and $\mu_{0}^{2}=m_{c}^{2}$, respectively, and $\mu_{0}^{2}=1 \mathrm{GeV}^{2}$ is considered for the gluon and light-quarks. In Tables V, VI, VII and VIII, we list all experimental data sets included in our global analysis, discussed in Sec. VA, and the $\chi^{2}$ values per degree of freedom pertaining to the LO and NLO fits are presented for each collaboration based on data points. Also the relative normalization calculated of fit for each data set is reported in these tables. Indeed the global minimization of $\chi^{2}$, discussed in Sec. IIIC , in the global fit considerably improves after taking into account relative normalization. The $\pi^{+}$and $K^{+}$fragmentation densities and their uncertainties are presented in Figs. 6] and 7 at $\mu_{0}^{2}=1 \mathrm{GeV}^{2}$ for the gluon and light-quarks and $\mu_{0}^{2}=m_{c}^{2}$ and $\mu_{0}^{2}=m_{b}^{2}$ for $c$ and $b$-quarks at NLO. We present the FFs uncertainties for $\Delta \chi^{2}=1$ and $\Delta \chi^{2}=22.43$ for pion and $\Delta \chi^{2}=24.58$ for kaon in Figs. 6] and 7. The method of error calcula-

tion is described in Secs. IIID and IIIE. 

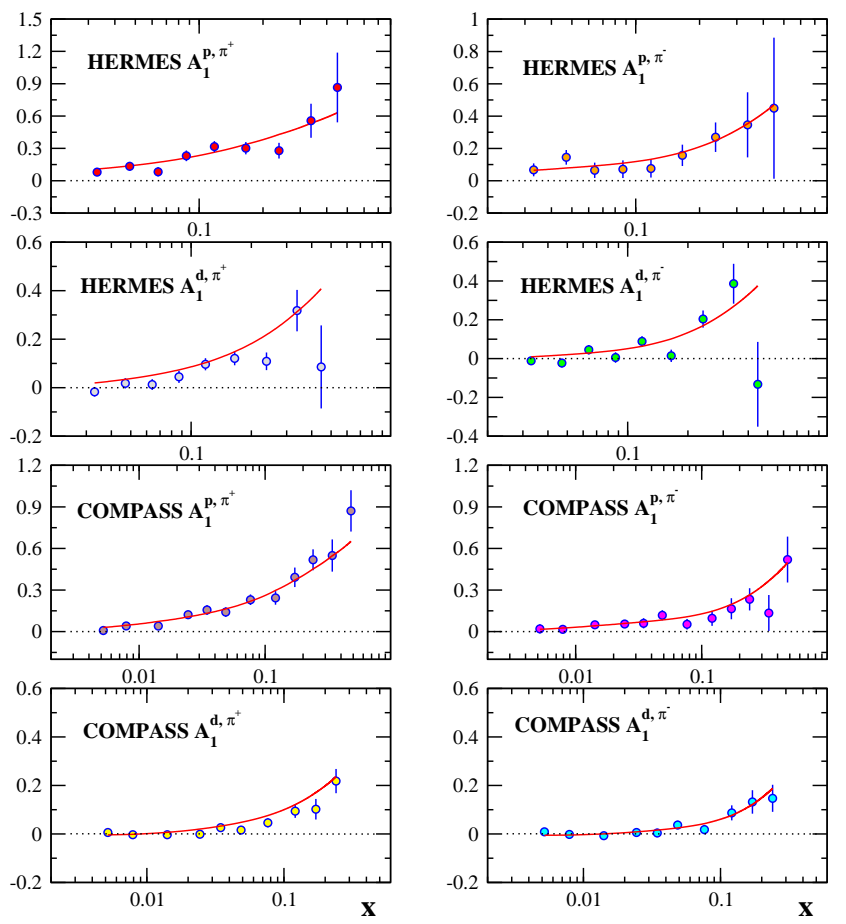

FIG. 4: Double spin asymmetry SIDIS data for $\pi^{+}$and $\pi^{-}$ from HERMES and COMPASS [25 27] at measured $x$ and $Q^{2}$ and comparison with the fit results of our global analysis at NLO.
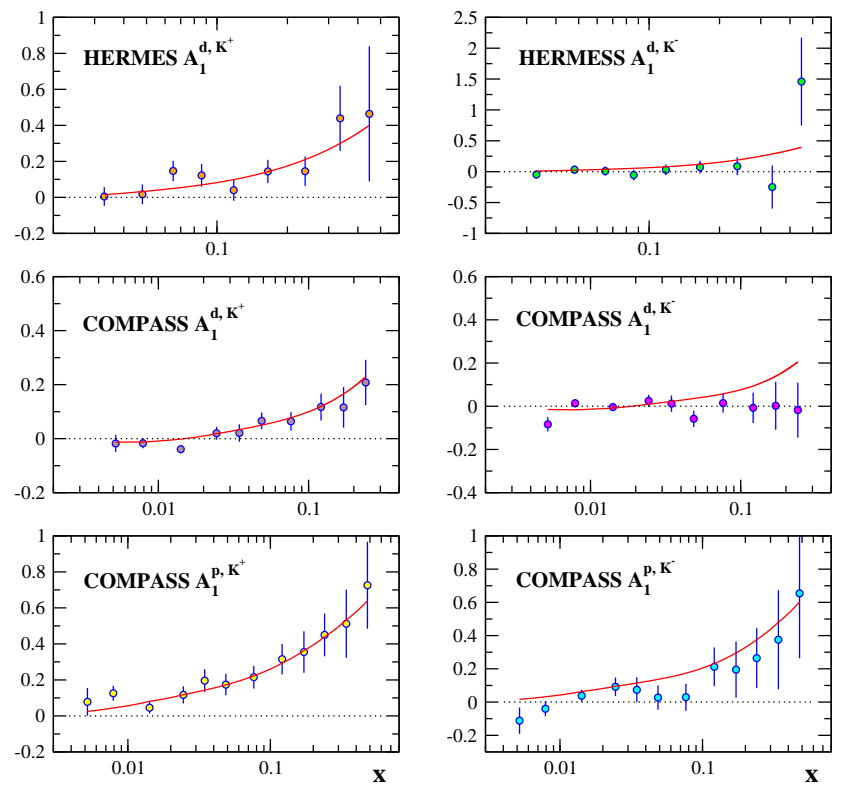

FIG. 5: Double spin asymmetry SIDIS data for $K^{+}$and $K^{-}$ from HERMES and COMPASS [25-27] at measured $x$ and $Q^{2}$ and comparison with the fit results of our global analysis at NLO.

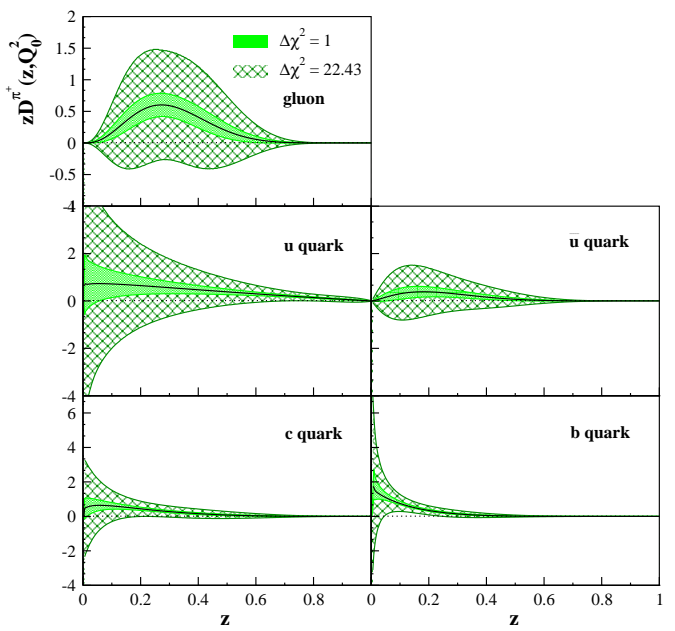

FIG. 6: Fragmentation densities and their uncertainties are shown for $\pi^{+}$at $Q_{0}^{2}=1 \mathrm{GeV}^{2}, m_{c}^{2}$ and $m_{b}^{2}$ at NLO. Their uncertainties are presented for $\Delta \chi^{2}=1$ (inner bands) and $\Delta \chi^{2}=22.43$ (outer bands).

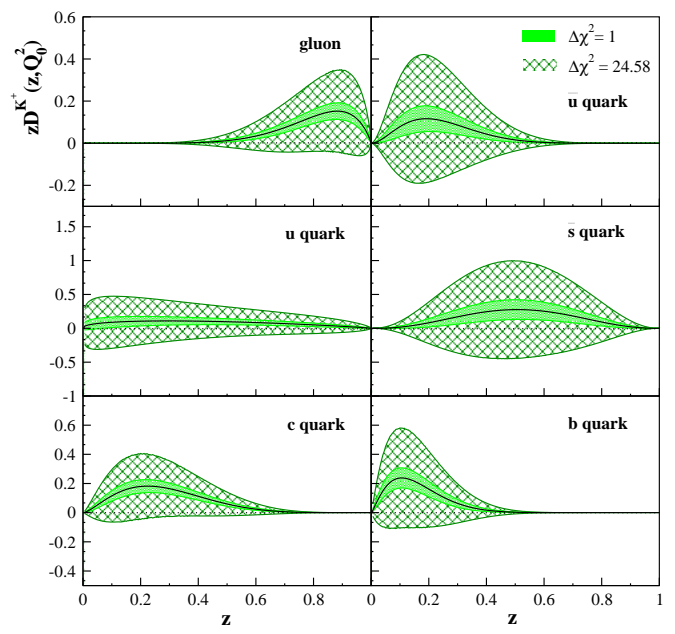

FIG. 7: Fragmentation densities and their uncertainties are shown for $K^{+}$at $Q_{0}^{2}=1 \mathrm{GeV}^{2}, m_{c}^{2}$ and $m_{b}^{2}$ at NLO. Their uncertainties are presented for $\Delta \chi^{2}=1$ (inner bands) and $\Delta \chi^{2}=24.58$ (outer bands).

ysis affect the results, we applied one of the most accurate PPDFs, i.e. KATAO PPDFs [35] which are obtained from a global analysis of DIS data. The comparison of extracted pion and kaon fragmentation functions by including KATAO 35] and DSSV [3] PPDFs are shown in Figs. 8 and 9 at NLO. As it is seen the differences are small and negligible and these differences become smaller by increasing the energy scaling. Therefore, the different choices of PPDFs do not change our result considerably. Since we would like to present how much the asymmetry SIDIS data are effective for determination of FFs, 
in Figs 10 and 11 the FFs for different flavors are presented in two cases at $\mu^{2}=M_{Z}^{2}$. In the first case we determine FFs by fitting on the single-inclusive electronpositron annihilation (SIA) and also SIDIS asymmetry data. According to the last section, we assume asymmetry between valence or favored fragmentation functions and unfavored fragmentation functions for both pion and kaon because the possibility of $\pi^{+} / K^{+}$-production from valence or favored quarks is more than sea or unfavored quarks. Moreover, SIDIS data help us to specify the difference between the quark and anti-quark distributions

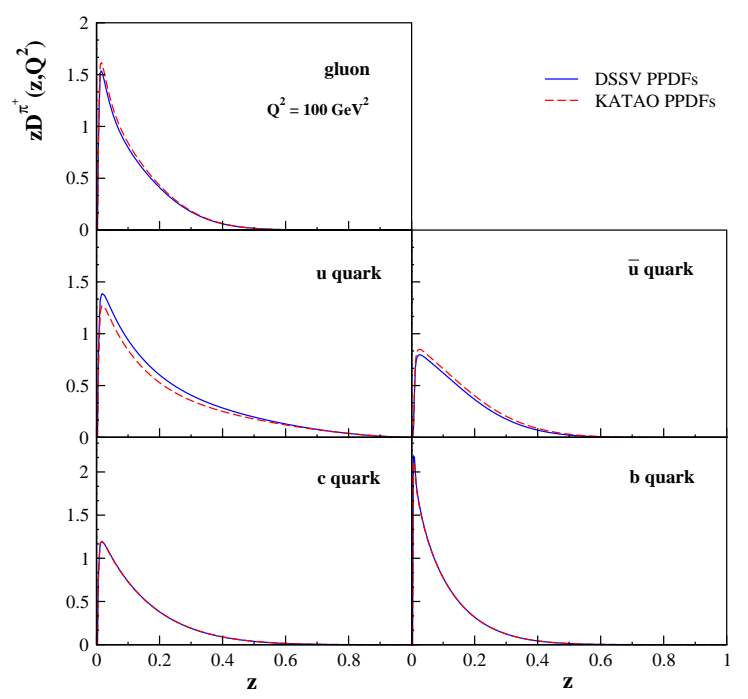

FIG. 8: Comparison of extracted pion fragmentation functions at $Q^{2}=100 \mathrm{GeV}^{2}$ using KATAO 35] and DSSV 3] PPDFs at NLO.

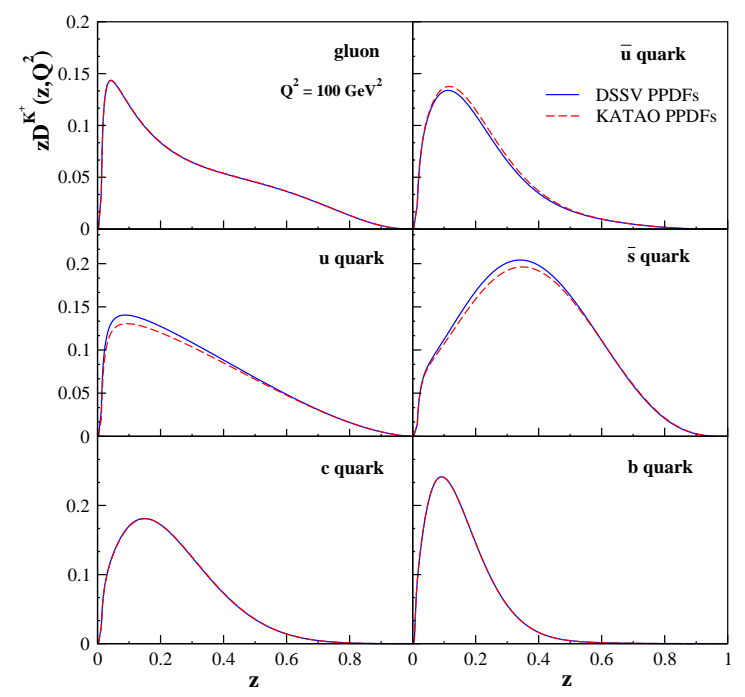

FIG. 9: Comparison of extracted kaon fragmentation functions at $Q^{2}=100 \mathrm{GeV}^{2}$ using KATAO [35] and DSSV [3] PPDFs at NLO.

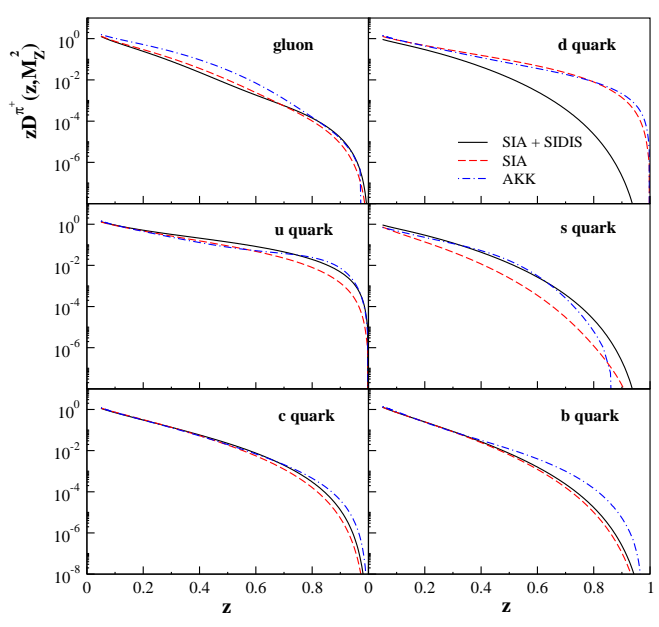

FIG. 10: Comparison of calculated $\pi^{+}$FFs at $Q^{2}=M_{Z}^{2}$ from fitting on SIA data $8-17$ with (solid lines) and without (dashed lines) SIDIS data 25 27] at NLO. The results are compered with AKK [28] (dot-dashed lines) too.

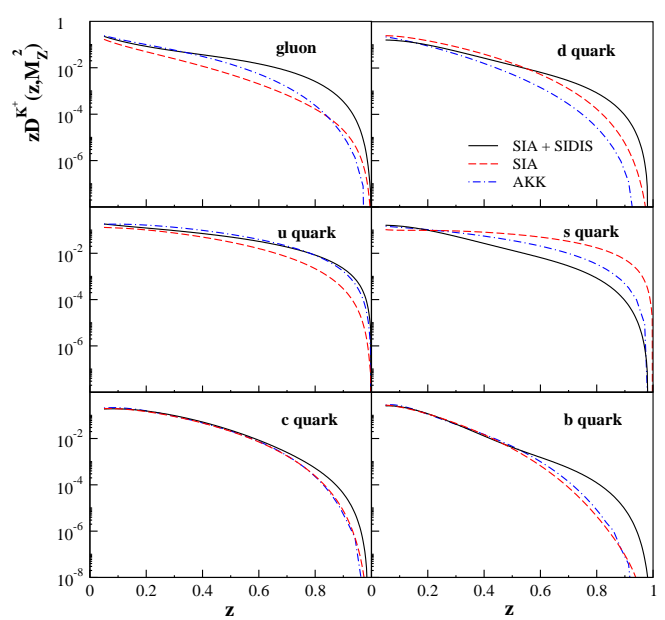

FIG. 11: Comparison of calculated $K^{+}$FFs at $Q^{2}=M_{Z}^{2}$ from fitting on SIA data [8 17] with (solid lines) and without (dashed lines) SIDIS data [25 27] at NLO. The results are compered with AKK 28] (dot-dashed lines) too.

in the nucleon considering outgoing produced hadrons which is not possible in fully inclusive experiments.

In the second case we calculate FFs by fitting just on the single-inclusive electron-positron annihilation (SIA) data. Since we omit asymmetry SIDIS data from our fit, the symmetry between the quark and anti-quark is assume in this case

$$
\begin{aligned}
& D_{u}^{H}\left(z, \mu_{0}^{2}\right)=D_{\bar{u}}^{H}\left(z, \mu_{0}^{2}\right), \\
& D_{d}^{H}\left(z, \mu_{0}^{2}\right)=D_{\bar{d}}^{H}\left(z, \mu_{0}^{2}\right), \\
& D_{s}^{H}\left(z, \mu_{0}^{2}\right)=D_{\bar{s}}^{H}\left(z, \mu_{0}^{2}\right) .
\end{aligned}
$$




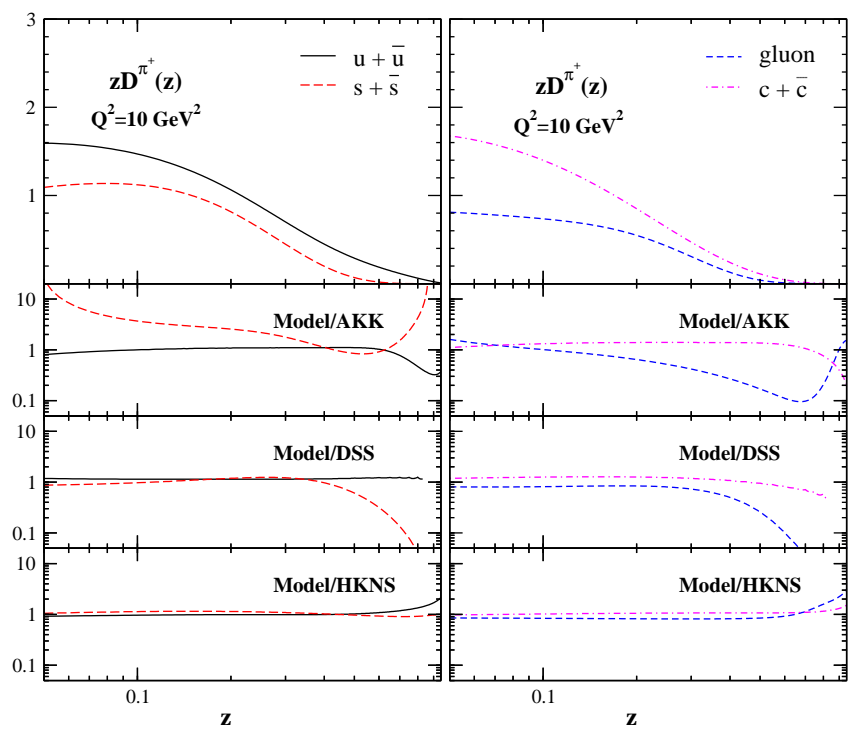

FIG. 12: Upper panels: fragmentation densities for $\pi^{+}$at $Q^{2}=10 \mathrm{GeV}^{2}$ at NLO. Rest panels: ratios of our fragmentation densities to the ones of HKNS, DSS and AKK [23, 24, 28].

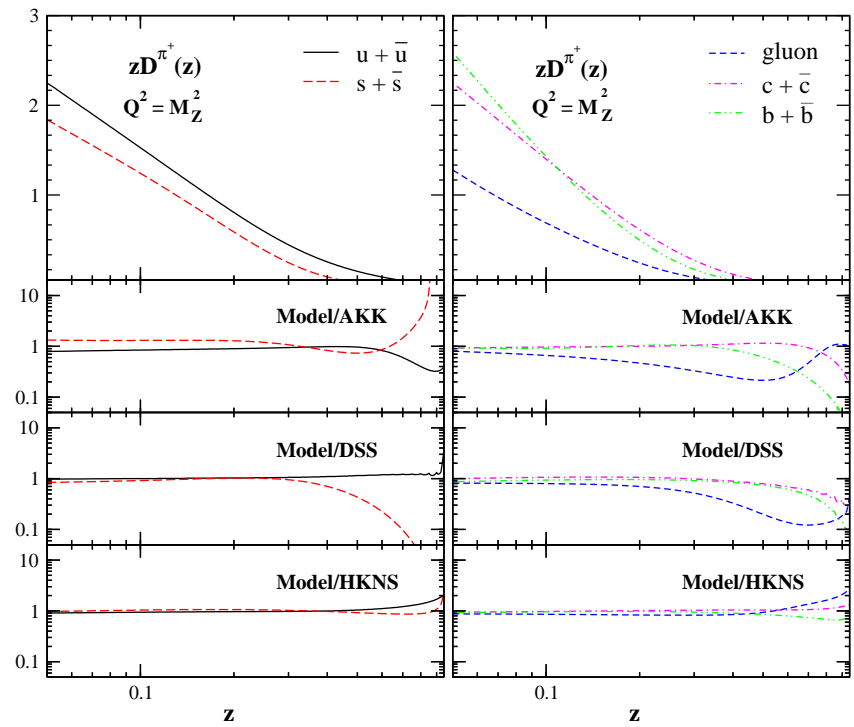

FIG. 13: Upper panels: fragmentation densities for $\pi^{+}$at $Q^{2}=M_{Z}^{2}$ at NLO. Rest panels: ratios of our fragmentation densities to the ones of HKNS, DSS and AKK [23, 24, 28].

According to Figs 10 and 11, the SIDIS data are effective on different partons of FFs. Also our results are compered with AKK model in these Figs.

We also present the $\pi^{+}$and $K^{+}$fragmentation densities at the scales $\mu^{2}=10 \mathrm{GeV}^{2}$ and $\mu^{2}=M_{Z}^{2}$ and the ratios of our fragmentation densities to the ones presented by HKNS, DSS and AKK [23, 24, 28] are shown in Figs. 12, 13, 14 and 15, According to these figures, our FF models densities are different in comparison with other models at large $z$ and it is not unexpected because according to Figs. 10 and 11, the SIDIS data impression on the FFs at large $z$ is more than small $z$ then the ratios in Figs. 12 , 13, 14 and 15 are mush better agreement at small $z$. Although when $Q^{2}$ increases the difference between models decreases.

Since, unlike our assumption, the light-quarks functional forms are separated in the AKK analysis due to fully flavor separated OPAL data, then the difference between 


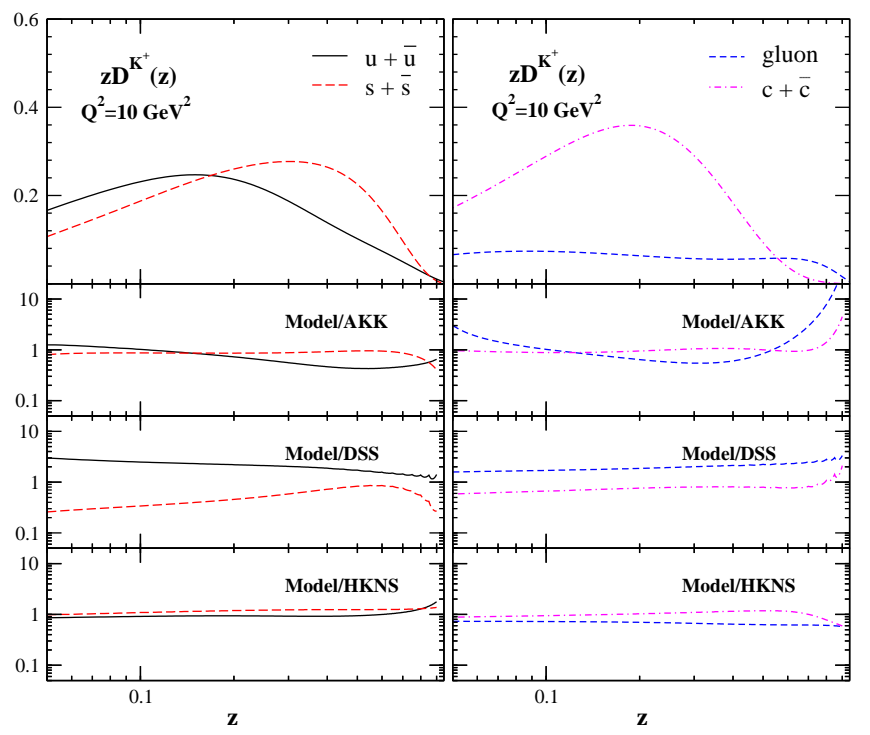

FIG. 14: Upper panels: fragmentation densities for $K^{+}$at $Q^{2}=10 \mathrm{GeV}^{2}$ at NLO. Rest panels: ratios of our fragmentation densities to the ones of HKNS, DSS and AKK [23, 24, 28].

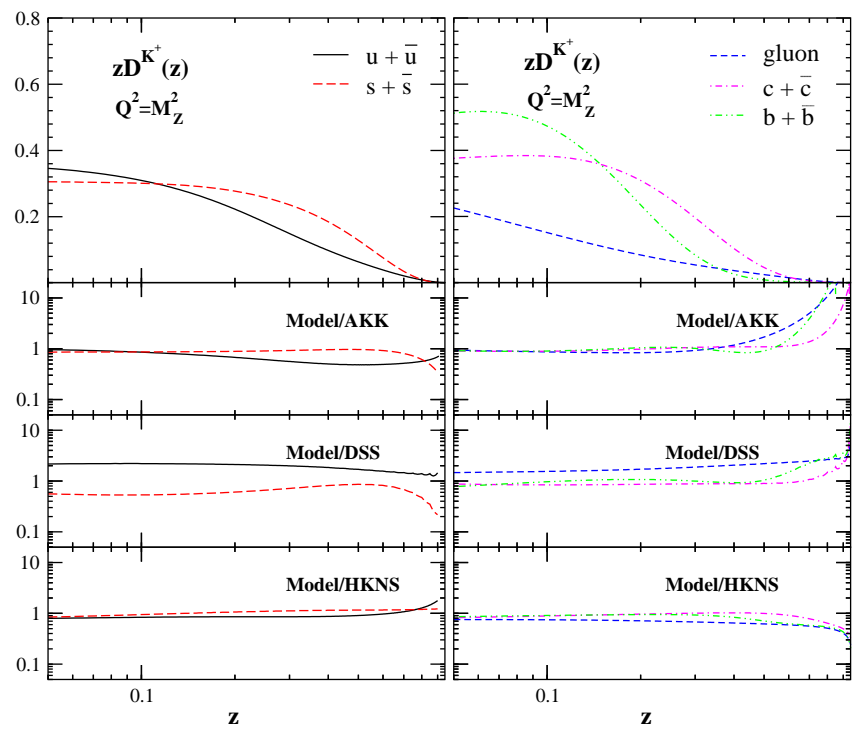

FIG. 15: Upper panels: fragmentation densities for $K^{+}$at $Q^{2}=M_{Z}^{2}$ at NLO. Rest panels: ratios of our fragmentation densities to the ones of HKNS, DSS and AKK [23, 24, 28].

our FFs results and AKK results is more than DSS and HKNS in Figs. 12, 13, 14 and 15, These data which are not used in the all analysis, such as HKNS, are more difficult to appreciate within purturbative QCD beyond the LO. Then we just use of untagged OPAL data and also the light-quarks functions are not separated in our analysis such as DSS and HKNS analysis.

\section{Quadratic behavior of $\Delta \chi^{2}$}

According to the Hessian method which is discussed in IIID we want to indicate if $\Delta \chi^{2}$ shows the assumed quadratic behavior on the parameters from the best fit. To explore this further, we present the dependance of $\Delta \chi^{2}$ global along some random samples of eigenvector directions to illustrate the deviations of the $\Delta \chi^{2}$ function from the expected quadratic dependence. As can be 
seen, to exhibit the quadratic approximation in Eq. 23 , Fig. 16] and Fig. 17 are presented to show the pion and kaon $\Delta \chi^{2}$ global along some random samples of eigenvector directions. Since the variation range of fitted term $\left[1-e^{-\gamma_{i} z}\right]$ for heavy partons $c / \bar{c}$ and $b / \bar{b}$ in global analysis of kaon (see Tables [II] and [V] and it improves the quadratic behavior of $\Delta \chi^{2}$. More details about the kaon parameters are explained in Sec. IIIB.

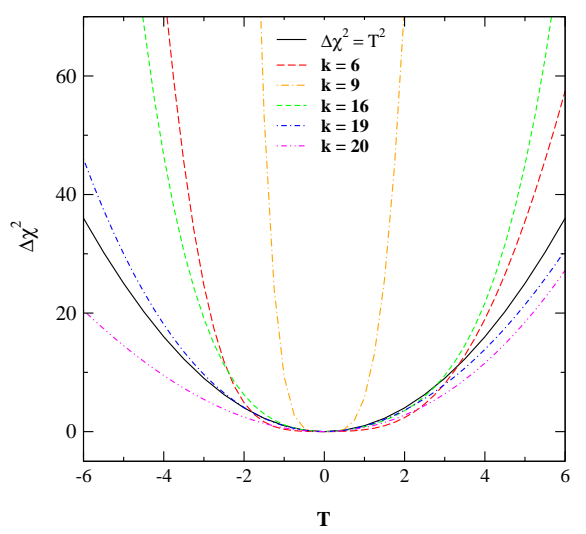

FIG. 16: Examples of pion $\Delta \chi^{2}$ deviations from the expected quadratic behavior $\Delta \chi^{2}=T^{2}$ for random sample eigenvector directions.

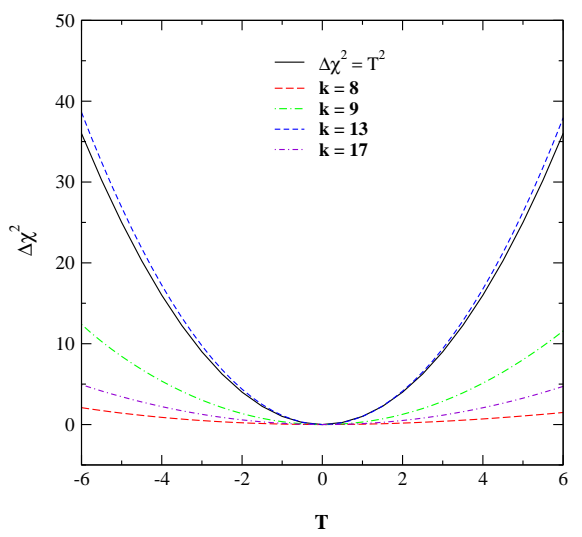

FIG. 17: Examples of kaon $\Delta \chi^{2}$ deviations from the expected quadratic behavior $\Delta \chi^{2}=T^{2}$ for random sample eigenvector directions.

parameters is correlated, here only one of the parameters is varied. Fig. 16 presents pion $\Delta \chi^{2}$ along some random samples of eigenvector directions and eigenvalues, $k=6,9,16,19$ and 20 . In this figure the curve with the eigenvectors direction $k=19$ for pion shows the most idealistic quadratic behavior and some other curves with $k=6,9$ a deviation from the ideal parabolic behavior curve $\Delta \chi^{2}=T^{2}$. Also Fig. [17 presents kaon $\Delta \chi^{2}$ along some random samples of eigenvector directions and eigenvalues, $k=8,9,13$ and 17 . To have a best fit we omit the
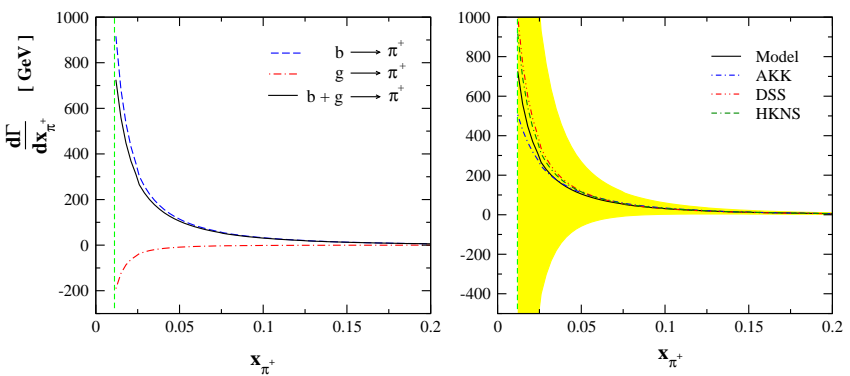

FIG. 18: Left panel: Energy distribution of $\pi^{+}$-meson in top decay considering the fragmentation contribution of b-quark (dashed line) and gluon (dot-dashed line) into the $\pi^{+}$and the total contribution (solid line) at $\mu_{F}=m_{t}$. Right panel: Energy distribution and its uncertainty of $\pi^{+}$considering the FFs obtained by our model, AKK, DSS and HKNS 23, 24, 28].
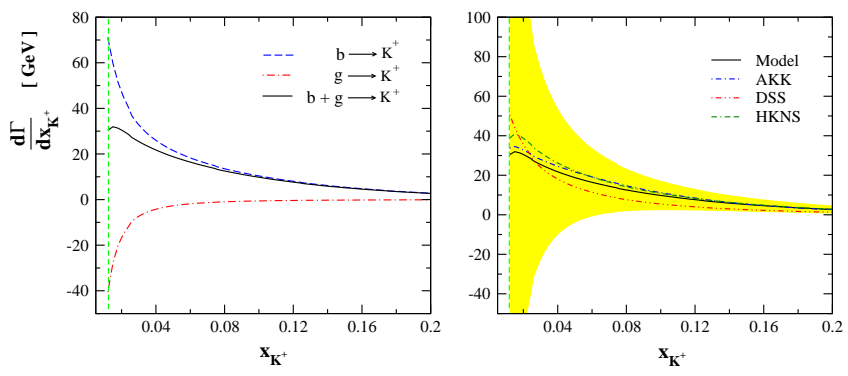

FIG. 19: Left panel: Energy distribution of $K^{+}$-meson in top decay considering the fragmentation contribution of b-quark (dashed line) and gluon (dot-dashed line) into the $K^{+}$and the total contribution (solid line) at $\mu_{F}=m_{t}$. Right panel: Energy distribution and its uncertainty of $K^{+}$considering the FFs obtained by our model, AKK, DSS and HKNS 23, 24, 28].

\section{Energy spectrum of light mesons in top-quark decay}

Now, by having the pion and kaon fragmentation functions in every scale, we make our phenomenological prediction for energy spectrum of light mesons in top decay. 
Therefor we adopt from Ref. 60 the input parameter values $G_{F}=1.16637 \times 10^{-5} \mathrm{GeV}^{-2}, m_{t}=172.0 \mathrm{GeV}$, and $m_{W^{+}}=80.399 \mathrm{GeV}$. In Figs. 18 and 19, we show our predictions for the size of the NLO corrections and their uncertainties, by comparing the relative importance of the $b \rightarrow \pi^{+} / K^{+}$(dashed line) and $g \rightarrow \pi^{+} / K^{+}$(dotdashed line) fragmentation, on a logarithmic scale. As it is seen the gluon fragmentation leads to an appreciable reduction in decay rate at low- $x_{H}$ region and for higher values of $x_{H}$, the $b \rightarrow H$ contribution is dominant. The mass of light meson is responsible for the appearance of the threshold at $x_{H}=2 m_{H} /\left(m_{t}^{2}-m_{W}^{2}\right)$. For comparison, we also show the energy spectrum of light mesons in top decay using the FFs obtained by AKK, DSS and HKNS collaborations in Figs. 18 and 19.

\section{CONCLUSIONS}

We have determined the non-perturbative parton fragmentation functions for pion and kaon at LO and NLO approximation from global analysis of single-inclusive electron-positron annihilation $e^{+} e^{-} \rightarrow(\gamma, Z) \rightarrow H+X$ and double spin asymmetry from semi-inclusive deep inelastic scattering data $A_{1}^{N, H}, \vec{l}(l)+\vec{N} \rightarrow l^{\prime}\left(l^{\prime}\right)+H+X$. Our analysis was based on zero mass variable flavor number scheme (ZM-VFNS) where all quarks are treated as massless particles. Our new parameterization form covers a wide kinematic range $z$ because of the extra term $\left[1-e^{-\gamma_{i} z}\right]$ which controls medium $z$ region and improves the accuracy of the global fit. Figs. 1, 2, 3, 4, and 5 show the comparison of our model with SIA and double spin asymmetry SIDIS experimental data and indicate that our model is successful. We determined the FFs of gluon and light-quarks at the initial scale $\mu_{0}^{2}=1 \mathrm{GeV}^{2}$ and the FFs of heavy quarks at $\mu_{0}^{2}=m_{c}^{2}$ and $\mu_{0}^{2}=m_{b}^{2}$. Evaluation determined by the DGLAP equations. The theoretical results of $b$ heavy quark for pion in our model and other models such as HKNS and DSS [23, 24] deviate from the SLD and DELPHI data at large $z$ and any deviance between theory and experimental data occurs a large $\chi^{2}$. In comparison with other group we applied, for the first time, spin asymmetry data $\left(A_{1}^{N, H}\right)$ in the global analysis of the fragmentation functions and the energy scales which are reported for the SIDIS experimental data are low energy scales which are usually smaller than the $e^{+} e^{-}$annihilation scales (see Tables V, VI, VII and VIII). On the other hand adding the SIDIS data in a global fit leads us to test the universality of parton fragmentation functions so that the results are in a good agreement with the FFs of other models. We also used one of the most accurate polarized and unpolarized parton distribution functions, i.e. NLO DSSV for polarized PDFs and NLO KKT12 for unpolarized PDFs. Using PDFs to determine FFs both indicates the universality of PDFs and is a good test for perturbative QCD analysis. We can also apply fragmentation functions to determine parton distribution into the proton, deuteron and neu- tron and show that parton densities do not depend on the corresponding cross sections and are universal. At the end we used the fragmentation functions to predict the energy spectrum of $\pi^{+}$and $K^{+}$mesons produced in top-quark decay. Comparison of our results for pion and kaon energy spectrum with other models shows that the fragmentation functions are universal, see Figs. 18 and 19.

\section{Acknowledgments}

We warmly acknowledge F. I. Olness, S. J. Brodsky and G. Kramer for valuable discussions, critical remarks and reading the manuscript. We appreciate E. Christova and M. Leitgab for useful suggestions and comments. A. N. K. thanks the SITP (Stanford Institute for Theoretical Physics) for partial support and the Physics Department of SMU (Southern Methodist University) for their hospitality during the completion of this work. A. N. K. and M. M. thank the CERN TH-PH division for its hospitality where a portion of this work was performed. We thank the School of Particles and Accelerators, Institute for Research in Fundamental Sciences (IPM) for financial support.

\section{Appendix: FORTRAN-code}

A FORTRAN package containing our un-
polarized fragmentation functions for pion
and kaon at LO and NLO can be found in
http://particles.ipm.ir/links/QCD.htm 73 or
obtained via e-mail from the authors. These functions
are interpolated using cubic splines in $Q^{2}$ and a linear
interpolation in log $\left(Q^{2}\right)$. The package includes an
example program to illustrate the use of the routines.


[1] V. N. Gribov and L. N. Lipatov, Sov. J. Nucl. Phys. 15, 438 (1972) [Yad. Fiz. 15, 781 (1972)]; G. Altarelli and G. Parisi, Nucl. Phys. B126, 298 (1977); Yu. L. Dokshitzer, Sov. Phys. JETP 46, 641 (1977) [Zh. Eksp. Teor. Fiz. 73, 1216 (1977)].

[2] D. de Florian, G. A. Navarro and R. Sassot, Phys. Rev. D 71, 094018 (2005) hep-ph/0504155.

[3] D. de Florian, R. Sassot, M. Stratmann and W. Vogelsang, Phys. Rev. D 80, 034030 (2009) arXiv:0904.3821 [hep-ph]].

[4] S. Kretzer, E. Leader and E. Christova, Acta Phys. Polon. B 33, 3743 (2002) hep-ph/0209055.

[5] S. Kretzer, E. Leader and E. Christova, Eur. Phys. J. C 22, 269 (2001) hep-ph/0108055.

[6] X. Artru, arXiv:1001.1061 [hep-ph].

[7] X. Artru and J. Czyzewski, Acta Phys. Polon. B 29, 2115 (1998) hep-ph/9805463.

[8] D. Buskulic et al. (ALEPH collaboration), Z. Phys. C66, 355 (1995); R. Barate et al., Phys. Rep. 294, 1 (1998).

[9] P. Abreu et al. (DELPHI collaboration), Eur. Phys. J. C5, 585 (1998).

[10] P. Abreu et al. (DELPHI collaboration), Nucl. Phys. B444, 3 (1995).

[11] R. Akers et al. (OPAL collaboration), Z. Phys. C63, 181 (1994).

[12] K. Abe et al. (SLD collaboration), Phys. Rev. D69, 072003 (2004).

[13] W. Braunschweig et al. (TASSO collaboration), Z. Phys. C42, 189 (1989).

[14] H. Aihara et al. (TPC collaboration), Phys. Rev. Lett. 52, 577 (1984); 61, 1263 (1988).

[15] R. Itoh et al. (TOPAZ collaboration), Phys. Lett. B345, 335 (1995).

[16] J. P. Lees et al. [BaBar Collaboration], arXiv:1306.2895 [hep-ex].

[17] M. Leitgab et al. [Belle Collaboration], arXiv:1301.6183 [hep-ex].

[18] D. P. Anderle, F. Ringer and W. Vogelsang, arXiv:1307.0701 [hep-ph].

[19] A. Jain, M. Procura, B. Shotwell and W. J. Waalewijn, arXiv:1207.4788 [hep-ph].

[20] Studies on fragmentation functions are listed in http://www.pv.infn.it/ radici/FFdatabase/.

[21] B. A. Kniehl, G. Kramer and B. Potter, Nucl. Phys. B 582, 514 (2000); B 597, 337 (2001); J. Binnewies, B. A. Kniehl and G. Kramer, Z. Phys. C 65, 471 (1995); Phys. Rev. D 52, 4947 (1995); 53, 3573 (1996); S. Albino, B. A. Kniehl and G. Kramer, Nucl. Phys. B 725, 181 (2005); B 734, 50 (2006); B. A. Kniehl and G. Kramer, Phys. Rev. D 71, 094013 (2005); 74, 037502 (2006).

[22] S. Kretzer, Phys. Rev. D 62, 054001 (2000) arXiv:hep-ph/0003177.

[23] M. Hirai, S. Kumano, T. -H. Nagai and K. Sudoh, Phys. Rev. D 75, 094009 (2007) hep-ph/0702250.

[24] D. de Florian, R. Sassot and M. Stratmann, Phys. Rev. D 75, 114010 (2007) hep-ph/0703242 [HEP-PH]].

[25] A. Airapetian et al. [HERMES Collaboration], Phys. Rev. D 71 (2005) 012003 arXiv:hep-ex/0407032.

[26] M. Alekseev et al. [COMPASS Collaboration], Phys. Lett. B 680, 217 (2009) arXiv:0905.2828 [hep-ex]].

[27] M. G. Alekseev et al. [COMPASS Collaboration], Phys.
Lett. B 693, 227 (2010) arXiv:1007.4061 [hep-ex]].

[28] S. Albino, B. A. Kniehl and G. Kramer, Nucl. Phys. B 803, 42 (2008) arXiv:0803.2768 [hep-ph]].

[29] R. K. Ellis, W. J. Stirling, and B. R. Webber, $Q C D$ and Collider Physics, Cambridge University Press (1996).

[30] J.C. Collins, Phys. Rev. D 58 (1998) 094002, arXiv:hep-ph/9806259

[31] B. A. Kniehl and G. Kramer, Phys. Rev. D 71, 094013 (2005) arXiv:hep-ph/0504058.

[32] J. Binnewies, B. A. Kniehl and G. Kramer, Z. Phys. C 65, 471 (1995) arXiv:hep-ph/9407347.

[33] T. Kneesch, B. A. Kniehl, G. Kramer and I. Schienbein, Nucl. Phys. B 799, 34 (2008) arXiv:0712.0481 [hep-ph]].

[34] H. Khanpour, A. N. Khorramian and S. A. Tehrani, J. Phys. G 40, 045002 (2013) arXiv:1205.5194 [hep-ph]].

[35] A. N. Khorramian, S. Atashbar Tehrani, S. Taheri Monfared, F. Arbabifar and F. I. Olness, Phys. Rev. D 83, 054017 (2011) arXiv:1011.4873 [hep-ph]].

[36] M. Gluck, E. Reya and A. Vogt, Eur. Phys. J. C 5, 461 (1998) arXiv:hep-ph/9806404.

[37] C. Pisano, Nucl. Phys. Proc. Suppl. 191, 35 (2009) arXiv:0812.3250 [hep-ph]].

[38] M. Gluck, C. Pisano and E. Reya, Eur. Phys. J. C 50, 29 (2007) arXiv:hep-ph/0610060.

[39] Y. Goto et al. [Asymmetry Analysis collaboration], Phys. Rev. D 62 (2000) 034017 arXiv:hep-ph/0001046; M. Hirai, S. Kumano and N. Saito [Asymmetry Analysis Collaboration], Phys. Rev. D 69 (2004) 054021 arXiv:hep-ph/0312112.

[40] E. Leader, A. V. Sidorov and D. B. Stamenov, arXiv:1007.4781 [hep-ph].

[41] D. de Florian, R. Sassot, M. Stratmann and W. Vogelsang, Phys. Rev. Lett. 101, 072001 (2008) arXiv:0804.0422 [hep-ph]].

[42] J. Blumlein and H. Bottcher, Nucl. Phys. B 841 (2010) 205 arXiv:1005.3113 [hep-ph]].

[43] E. Leader, A. V. Sidorov and D. B. Stamenov, Phys. Rev. D 82 (2010) 114018 arXiv:1010.0574 [hep-ph]].

[44] M. Hirai and S. Kumano [Asymmetry Analysis Collaboration], Nucl. Phys. B 813, 106 (2009) arXiv:0808.0413 [hep-ph]].

[45] A. N. Khorramian, A. Mirjalili and S. A. Tehrani, JHEP 0410 (2004) 062 arXiv:hep-ph/0411390.

[46] S. Atashbar Tehrani and A. N. Khorramian, JHEP 0707, 048 (2007) arXiv:0705.2647 [hep-ph]].

[47] A. N. Khorramian, S. Atashbar Tehrani, S. Taheri Monfared, F. Arbabifar and F. I. Olness, Phys. Rev. D 83 (2011) 054017 arXiv:1011.4873 [hep-ph]].

[48] S. Atashbar Tehrani, A. N. Khorramian, S. Taheri Monfared and F. Arbabifar, AIP Conf. Proc. 1374, 391 (2011).

[49] F. Arbabifar, A. N. Khorramian, S. Taheri Monfared and S. Atashbar Tehrani, Int. J. Mod. Phys. A 26, 625 (2011).

[50] S. Taheri Monfared, A. Khorramian, S. Atashbar Tehrani and Z. Haddadi, Nucl. Phys. Proc. Suppl. 210-211 (2011) 125.

[51] H. Khanpour and A. N. Khorramian, Acta Phys. Polon. B 41, 2929 (2010).

[52] S. Taheri Monfared, A. N. Khorramian, F. Arbabifar and S. Atashbar Tehrani, Acta Phys. Polon. B 41, 2921 (2010). 
[53] D. de Florian, M. Stratmann, and W. Vogelsang, Phys. Rev. D 57, 5811 (1998).

[54] A. A. Almasy, S. Moch and A. Vogt, Nucl. Phys. B 854, 133 (2012) arXiv:1107.2263 [hep-ph]].

[55] A. Mitov, S. Moch and A. Vogt, Phys. Lett. B 638, 61 (2006) hep-ph/0604053.

[56] S. Albino and E. Christova, Phys. Rev. D 81, 094031 (2010) arXiv:1003.1084 [hep-ph]].

[57] S. Albino, E. Christova and E. Leader, arXiv:1102.2305 [hep-ph].

[58] J. Binnewies, B.A. Kniehl, and G. Kramer, Phys. Rev. D 58, 034016 (1998); M. Cacciari and M. Greco, Nucl. Phys. B421, 530(1994).

[59] A. D. Martin, R. G. Roberts, W. J. Stirling and R. S. Thorne, Eur. Phys. J. C 28, 455 (2003) hep-ph/0211080.

[60] K. Nakamura et al. (Particle Data Group), J. Phys. G 37, 075021 (2010).

[61] M. Soleymaninia, A. Khorramian and M. Moosavi Nejad, J. Phys. Conf. Ser. 347, 012017 (2012).

[62] M. Soleymaninia, A. N. Khorramian and S. M. Moosavinejad, AIP Conf. Proc. 1492, 67 (2012).

[63] M. Hirai and S. Kumano, Prog. Theor. Phys. Suppl. 186, 244 (2010) arXiv:1008.3814 [hep-ph]].
[64] D. de Florian, R. Sassot and M. Stratmann, J. Phys. Conf. Ser. 110, 022045 (2008) arXiv:0708.0769 [hep-ph]].

[65] A. D. Martin, W. J. Stirling, R. S. Thorne and G. Watt, Eur. Phys. J. C 63, 189 (2009) arXiv:0901.0002 [hep$\mathrm{ph}]$.

[66] J. Pumplin, D. R. Stump and W. K. Tung, Phys. Rev. D 65, 014011 (2001) hep-ph/0008191.

[67] M. Epele, R. Llubaroff, R. Sassot and M. Stratmann, Phys. Rev. D 86, 074028 (2012) arXiv:1209.3240 [hep$\mathrm{ph}]$.

[68] J. Pumplin, D. Stump, R. Brock, D. Casey, J. Huston, J. Kalk, H. L. Lai and W. K. Tung, Phys. Rev. D 65, 014013 (2001) hep-ph/0101032.

[69] M. Hirai et al. [Asymmetry Analysis Collaboration], Phys. Rev. D 69, 054021 (2004) hep-ph/0312112.

[70] S. Moch, P. Uwer, Phys. Rev. D 78 (2008) 034003, arXiv:0804.1476 [hep-ph].

[71] N. Cabibbo, Phys. Rev. Lett. 10 (1963) 531; M. Kobayashi, T. Maskawa, Prog. Theor. Phys. 49 (1973) 652.

[72] B. A. Kniehl, G. Kramer and S. M. M. Nejad, Nucl. Phys. B 862, 720 (2012) arXiv:1205.2528 [hep-ph]].

[73] Program summary URL: http://particles.ipm.ir/links/QCD.htm 